\title{
Beitrag zur Geschichte plastischer Berechnungsmethoden im Stahlbau
}

\author{
Jacques Heyman gewidmet
}

Jacques Heyman (Bild 1b) leistete einen wesentlichen Beitrag zur Entwicklung der Baustatik im 20. Jahrhundert. Nach seinem Studium trat er dem berühmten Team unter der Leitung von John Fleetwood Baker (1901-1985) (Bild 1a) in Cambridge bei, das die Grundlagen für die Berechnung von Stahlskelettkonstruktionen nach der Plastizitätstheorie entwickelte. Baker hatte zwischen 1928 und 1936 für das Steel Structures Research Committee (SSRC) an der Entwicklung einer rationellen Methode für die Schnittkraftberechnung von Rahmenkonstruktionen nach der Elastizitätstheorie gearbeitet. Im Rahmen dieser Arbeiten konnten erstmals Versuche an ausgeführten Bauwerken durchgeführt werden. Die Ergebnisse waren überraschend, denn entgegen der vorherrschenden Meinung stellte sich heraus, daß die Schnittkraftberechnung nach der Elastizitätstheorie nicht geeignet war, den tatsächlichen Zustand von Stahlkonstruktionen vorherzusagen. Diese Einsicht veranlaßten Baker, eine andere Forschungsrichtung einzuschlagen. 1936 reiste Baker zur IVBH-Konferenz nach Deutschland, wo er Verbindung mit einer Reihe von Wissenschaftlern aus Mitteleuropa knüpfte, die sich bereits seit über zwei Jahrzehnten mit der Erforschung des elastoplastischen Verhaltens von Stahlträgern befaßt hatten. Nach seiner Rückkehr setzte er seine Forschungsarbeiten in diesem Bereich an der Universität von Bristol fort. Er wiederholte die Versuche von Hermann Maier-Leibnitz (1885-1962) und führte eine eigene Versuchsreihe an Rahmenkonstruktionen durch. Diese Arbeiten wurden durch den 2. Weltkrieg unterbrochen, konnten aber nach Kriegsende in Cambridge fortgesetzt werden und wurden durch die Bücher "The steel skeleton" [1], [2] sowie „Plastic design of portal frames" [3] gekrönt.

\section{Contribution to the history of plastic analysis methods in steel} construction. Jacques Heyman (Fig, (b) has been one of the main contributors to the development of structural theory in the 20th century. After graduating he joined the famous Cambridge Team directed by John Fleetwood Baker (1901-1985) (Fig. 1a) that established the basis for plastic analysis of steel frame structures. Baker had worked for the Steel Structures Research Committee (SSRC) between 1928 and 1936, with the specific task of writing a rational code for elastic analysis of frames. For the first time real structures were tested. The results were surprising; elastic analysis was not suitable for predicting the actual state of the steel structure, contradicting what was believed at the time. This insight caused Baker to change the direction of his research. In 1936 Baker traveled to Germany to attend the IABSE Conference, where he met those researchers from central Europe who, for over two decades, had been studying the elasto-plastic behaviour of steel beams. Back in the UK, he continued studying this field at Bristol University; he repeated Hermann Maier-Leibnitz's tests and started a program of tests on frames. The Second World War interrupted
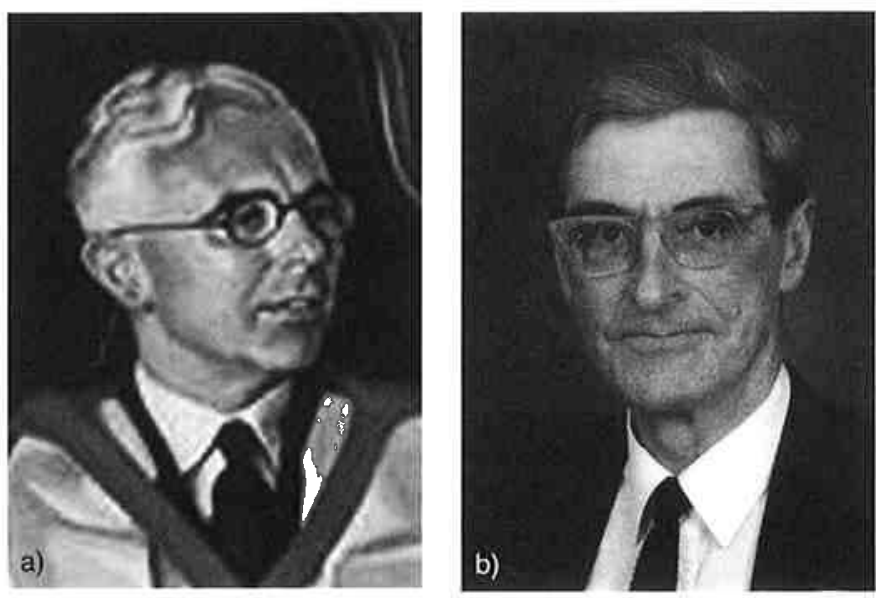

Bild 1. a) John Fleetwood Baker und b) Jacques Heyman Fig. 1. a) John Fleetwood Baker and b) Jacques Heyman

the work, which would continue at Cambridge once the war was over and crowned with success by the books "The steel skeleton" [1], [2] and "Plastic design of portal frames" [3].

\section{Vorbemerkungen}

Am 2. und 3. Dezember 2005 organisierte der zweitgenannte Verfasser am „Instituto Juan de Herrera“ der „Escuela Téchnica Superior de Arcquitectura de Madrid“ zu Ehren des britischen Bauingenieurs Jacques Heyman das Symposium "Historical perspectives on structural analysis“ [4]; damit erblickte die erste internationale Tagung zur Geschichte der Baustatik das Licht der Welt.

Im Rahmen seiner 1981 am Fachgebiet „Statik der Baukonstruktionen" derTU Berlin fertiggestellten Diplomarbeit zur geschichtlichen Entwicklung der Gewölbetheorie lernte der erstgenannte Verfasser die Arbeiten Heymans zur statischen Analyse historischer Gewölbe kennen; nach und nach entdeckte er auch die anderen bahnbrechenden Beiträge Heymans zur Baustatik, zur Geschichte der Baustatik und zum Stahlbau.

Heymans Liebe zur Stahlbauweise und Historiographic der Baustatik hat uns auf die Idee gebracht, aus Anlaß des 75. Jahrganges derZeitschrift STAHLBAU (die erste Nummer erschien am 6. April 1928), die großen Verdienste Heymans um den Fortschritt der Stahlbauweise exemplarisch im historischen Kontext zu würdigen. Neben den einschlä- 
gigen Arbeiten Heymans, konnten wir uns auf den o. g. Kongreßband [4], das Buch zur Geschichte der Baustatik [5] und ergänzenden Zeitschriftenaufsätzen - insbesondere aus den ersten Jahrgängen von STAHLBAU - abstützen.

\section{Von der Schlauheit des Baustahls}

Vor gar nicht allzu langer Zeit rühmten Baustatiker im Stahlbau immer dann die Schlauheit, die Selbsthilfe des Materials, wenn die Rechenergebnisse ihrer auf der Elastizitätstheorie basierenden baustatischen Modelle das reale Tragverhalten nicht adäquat widerspiegelten. Sie rühmten damit ihren bildsamen, duktilen Werkstoff Stahl, der über Tragreserven verfügt, die sich jenseits der Elastizitätsgrenze verbergen: zur Metapher geronnener metaphysischer Überschuß. Die mit Sicherheit vorgetragene Ruhmrede drückt Unsicherheit über die Quantifizierbarkeit der Schlauheit, der Selbsthilfe des Materials durch die Baustatik in der Akkumulationsphase der Baustatik (1900-1925) aus. Fand schon mit der Entwicklung der Theorie II. Ordnung auf der Seite des Zusammenhangs zwischen Belastungs- und Schnittkraftzustand die Brechung der Herrschaft des Linearen in der Inventionsphase der Baustatik (1925-1950) statt, so setzten im selben Zeitraum Wissenschaftler der Baustatik und Technischen Mechanik die linear-elastische Spannungs-Dehnungsbeziehung (Hookesches Gesetz) der Kritik der Wirklichkeit aus. Damit wurden zwei Breschen in die die klassische Baustatik kennzeichnende Trinität des Linearen (Statik, Material, Geometrie) geschlagen. Nichtlineare Dehnungs-Verschiebungsbeziehungen (Geometrie) dagegen entwickelten sich am Ende der Innovationsphase (1950-1975) zum wissenschaftlichen Gegenstand der Baustatik, um die damals aufkommenden leichten Flächentragwerke mit Computern zu berechnen.

\section{Erste Ansätze}

Den ersten Ansatz zur experimentellen Erfassung der Traglasten von Stahlträgern formulierte 1914 der ungarische Bauingenieur Gábor v. Kazinczy (1889-1964). Er untersuchte beidseitig eingespannte Träger mit der lichten Länge $\ell=\ell_{1}=5,60 \mathrm{~m}$ und $\ell=\ell_{2}=6 \mathrm{~m}$ in die jeweils ein Stahlprofil I 160 einbetoniert waren [6] (Bild 2). In Bild 3 ist $v$. Kazinczys statisches Modell nach einer Veröffentlichung von Heyman mit der Gleichstreckenlast w dargestellt [8.73, S. 128] ${ }^{1}$. Aus seinen Versuchsergebnissen schloß v. Kazinczy, daß beim beidseitig eingespannten Träger (zweifach statisch unbestimmt) drei Querschnitte fließen (Fließgelenke) müssen, um den Versagenszustand zu erreichen: Der Träger muß also nicht nach der elastischen Lösung - also auf das Biegemoment $w \cdot \ell^{2} / 12$ - bemessen werden (Bild $3 b$ ), sondern nur auf den Wert $w \cdot \ell^{2} / 16$ (Bild 3c).

1917 befaßte sich der Delfter Professor N. C. Kist mit dem Traglastproblem (s. [8, S. 34-36]); 1920 schlug er vor, Konstruktionen aus Flußeisen nach dem ideal-elastischen und ideal-plastischen Stoffgesetz zu bemessen [9, S. 428]. Dieses idcalisierte Stoffgesetz bildet noch heute die Grundlage der auf der Traglasttheorie fußenden Bemessungsverlahren im Stahlbau. Die Begriffe Traglasttheorie und Fließ-

1 Nil $8 . x$ werclen im Folgenden die unter Abschnitt 8 der Nr. $\mathrm{x}$ iulperiilurlen Veriffentlichungen Heymans bezeichnet.

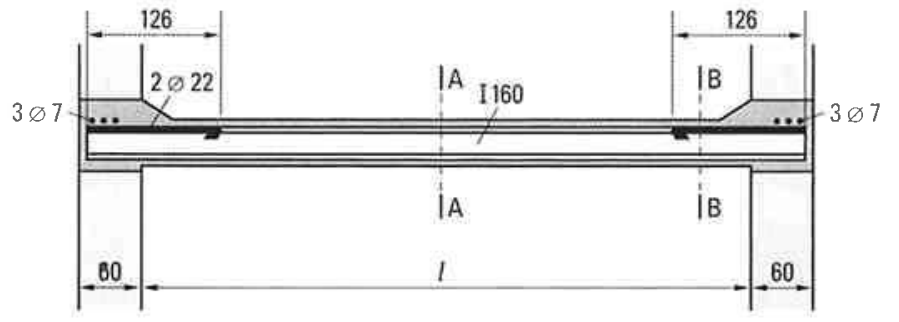

a)
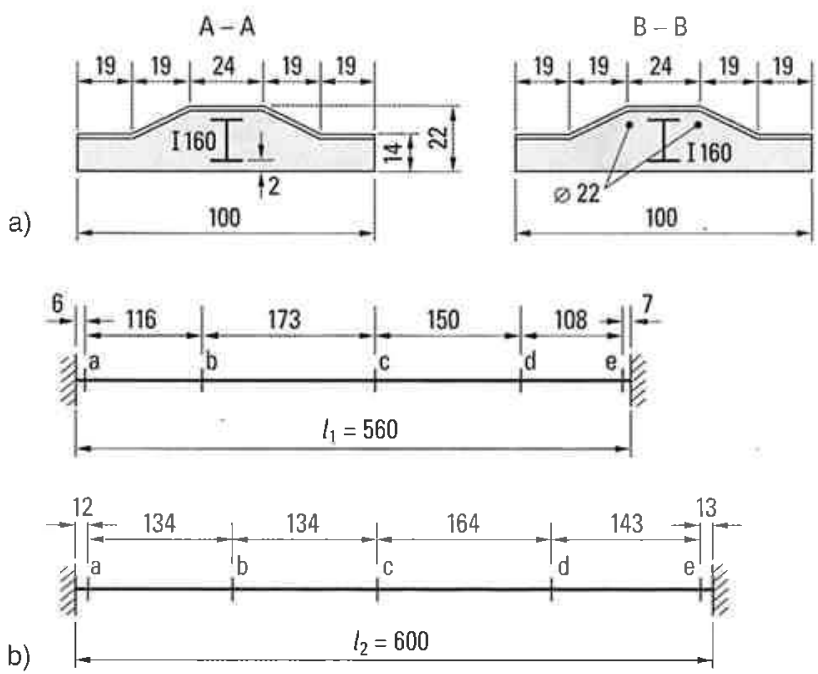

Bild 2. a) Kazinczys Versuchsträger; statische Systeme mit den Meßstellen $a, b, c, d$ und e für die Durchbiegung (Quelle: [7, S. 78]) (alle Maße in cm)

Fig. 2. a) Kazinczy's test beam; structural systems with points $a, b, c, d$ and $e$ for measuring the deflection (source: $[7$, p. 78]) (in $\mathrm{cm}$ )

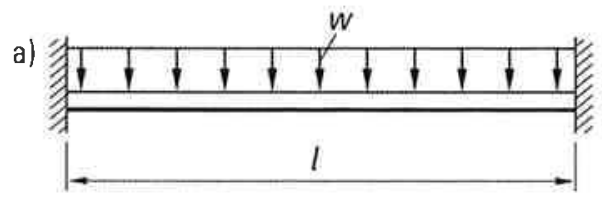

b)

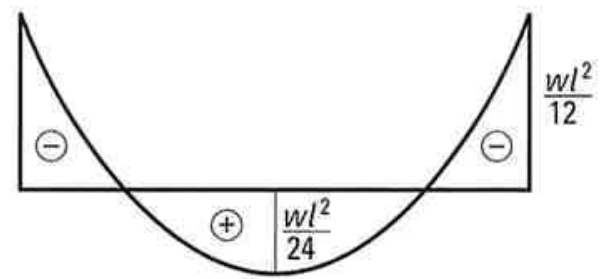

c)

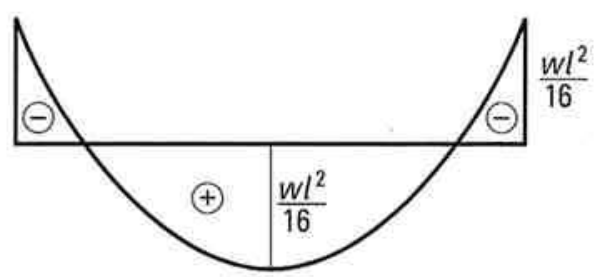

Bild 3. a) Beidseitig eingespannter Träger; b) elastische und c) plastische Lösung (Quelle: [8.73, S. 128])

Fig. 3. a) Fixed-ended beam; b) elastic and c) plastic solution (source: $[8.73, p .128]$ )

gelenktheorie werden im folgenden synonym gebraucht, obwohl der Begriff. Traglasttheorie der Oberbegriff ist ebenso verhält es sich mit den entsprechenden Kompositabildungen Traglastverfahren und Fließgelenkverfahren.

Die Einsicht, daß eine wirtschaftliche Bemessung von Stahlbauten allein auf der Grundlage der Elastizitätstheo- 
rie nicht mehr gegeben war, brach sich Ende der 1920er Jahre in Deutschland, Österreich, der Tschechoslowakei und dann in Großbritannien und der Sowjetunion Bahn. In Deutschland eröffneten Professor Martin Grüning (TH Hannover) auf theoretischer Ebene und Professor MaierLeibnitz (TH Stuttgart) auf experimenteller Ebene die Diskussion. Grüning erweiterte die Erkenntnisse v. Kazinczys über den plastischen Grenzzustand des beidseitig eingespannten Träger auf $\mathrm{n}$-fach statisch unbestimmte Systeme [10].

Maier-Leibnitz (s. [11]) brachte 1928 mit seinem Versuchsbericht über die tatsächliche Tragfähigkeit einfacher und durchlaufender Balkenträger aus Baustahl St 37 und aus Holz Licht in das Dunkel der Schlauheit des Materials: „Viele Konstrukteure, die im Eisenbau tätig sind, scheuen sich eingedenk der von Mohr und in anderen Lehrbüchern der Baustatik ausgesprochenen Bedenken vor der Anwendung des durchlaufenden Balkens und ziehen ihm den einfachen Balken und den Gelenkträger vor, obgleich bei diesen Trägerarten die erfahrenen Konstrukteuren schon lange bekannte Selbsthilfe des bildsamen Baustahls beschränkt ist (...). Um die Fragen zu klären, ob überhaupt der rechnerisch ermittelte Größtwert des Stützenmoments für die Tragfähigkeit durchlaufender Balken maßgebend ist und welchen Einfluß auf die Tragfähigkeit Stützensenkungen haben, ferner um zu zeigen, wie man wenigstens in einem Sonderfall die bisher nur gefühlsmäßig konstruktiv verwendete Selbsthilfe des Baustoffs ihrem Wesen nach erkennen und wie man sie bei der Querschnittsbemessung der Träger bewußt verwerten kann, ließ der Verfasser (...) die im folgenden (...) beschriebenen Versuche durchführen" [12, S. 11].

Maier-Leibnitz faßt seine Biegeversuche an Zweifeldträgern mit gleicher Spannweite dahingehend zusammen, daß kleinere Stützensenkungen keinen Einfluß auf die Tragfähigkeit haben und das maßgebende Bemessungsmoment nicht das Stützmoment, sondern ein Feldmoment sei, das betragsmäßig $75 \%$ des Stützmoments ist. Otto Mohr hatte schon 1860 die ungünstige Auswirkung von Stützensenkungen von Durchlaufträgern auf die Schnittkräfte quantifiziert [13] und damit die Bedenken gegen die Verwendung des Durchlaufträgers zahlenmäßig belegt. Aber Mohr legte seinen Überlegungen die Elastizitätstheorie zugrunde. Auf den Beitrag von Maier-Leibnitz gingen der Schriftleitung von „Die Bautechnik“" zahlreiche Zuschriften prominenter Bauingenieure ein, die im selben Jahrgang in Heft 20 publiziert wurden: Grüning und Kulka, Bohny,

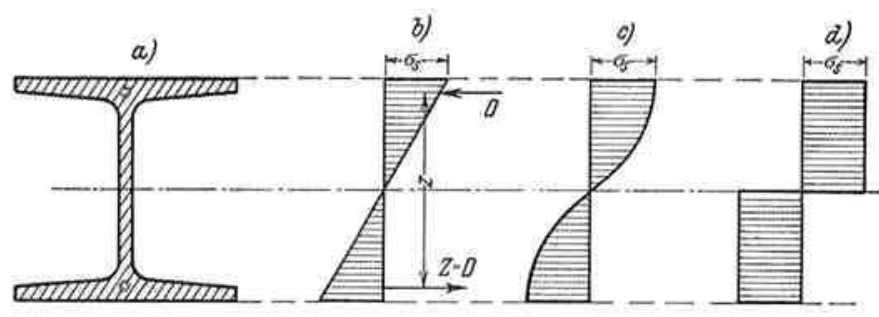

Bild 4. Spannungsverteilung nach Maier-Leibnitz; a) Querschnitt, b) elastischer Grenzzustand, c) teilplastizierter und d) vollplastizierter Querschnitt (Quelle: [14, S. 314])

Fig. 4. Stress distribution acc. to Maier-Leibnitz; a) cross section, b) elastic limit state, c) partially plastic and d) fully plastic cross section (source: [14, p. 314])
Metzler, Beyer, Jagschitz, Gaber, Krabbe, Bernhard sowie Lienau. 1929 schließlich interpretierte Maier-Leibnitz seine Traglastversuche mit eingespannten und einfachen I-Trägern aus St 37 vor dem Hintergrund des von Kist vorgeschlagenen ideal-elastischen-ideal-plastischen Stoffgesetzes und gab die Spannungsverteilung im vollplastizierten Querschinitt an (Bild 4).

\section{Grundlegung des Fließgelenkverfahrens}

Nun erscheinen in rascher Folge Aufsätze in Zeitschriften wie „Die Bautechnik“, „Der Bauingenieur“, „Der Stahlbau“ und der „Zeitschrift für Angewandte Mathematik und Mechanilk (ZAMM)" über die Berücksichtigung der Plastizität bei der Berechnung statisch unbestimmter Stahlkonstruktionen. Die wichtigsten Arbeiten stammen von Professor Josef Fritsche (TH Prag) und Karl Girkmann (Fa. WaagnerBiro, Wien). Während Fritsche 1930 den statisch bestimmten Einfeldträger, den beidseitig eingespannten Einfeldträger und spezielle Durchlaufträger untersucht [15] gibt Girkmann 1931/32 ein auf Versuchen abgestütztes plastisches Berechnungsverfahren für Rahmentragwerke an [16], [17].

\subsection{Josef Fritsche}

Fritsche entwickelt erstmals die Formeln für das Biegemoment $\mathrm{M}_{\mathrm{p} \ell}$ des vollplastizierten Rechteckquerschnitts und I-Querschnitts im Falle reiner Biegung [15, S. 852-855]. Nachdem er die erweiterte Differentialgleichung für die Balkenbiegung an der Berechnung der elastisch-plastischen Verformung eines Einfeldbalkens exemplifiziert, wendet sich Fritsche der Analyse statisch unbestimmter Systeme zu. Dieselbe Differentialgleichung löst er nun für den Fall des beidseitig eingespannten Balkens unter mittiger Einzellast; im vollplastizierten Zustand entstehen gleichzeitig Fließgelenke an den Einspannstellen und in Trägermitte (Bild 5).

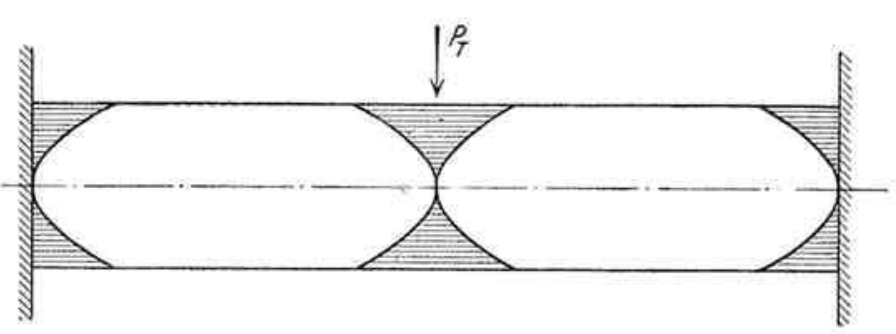

Bild 5. Fließgelenke des beidseitig eingespannten Balkens nach Fritsche (Quelle: [15, S. 873])

Fig. 5. Plastic hinges of a fixed-ended beam acc. to Fritsche (source: $[15, p .873]$ )

Dasselbe Verfahren wendet Fritsche zur Bestimmung der Traglast von Zwei- und Dreifeldträgern an. Fritsche schreibt: „Wie die bisherigen Beispiele zeigen, ist die Berechnung der Tragfähigkeit recht einfach, vermeidet die oft langwierige Berechnung der statisch unbestimmten Größen, obwohl die Kenntnis der rein elastischen Lösung, allerdings nur in allgemeiner Form, sehr vorteilhaft ist. Es liegt daher im Bereiche der Möglichkeit, für die eisernen Tragwerke des Hochbaues, wo Träger aus einfachen Walzeisen recht häufig sind, die Bemessung statt nach einer zulässigen Inanspruchnahme nach dem auf die beschriebene Weise ermittelten Tragvermögen durchzuführen" [15, 
S. 890]. Sein Verfahren der elastisch-plastischen Analyse von stählernen Balkentragwerken verifiziert Fritsche durch die Nachrechnung der Versuche von E. Meyer, H. MaierLeibnitz und J. H. Schaim. Fritsche kommt zum Schluß, „daß die Ungleichmäßigkeit der Höhe der Fließgrenze des Stahles für eine genaue rechnungsmäßige Überprüfung derselben ein beträchtliches Hindernis ist; doch zeigt sie, daß die Fehler, in Prozent der Tragfähigkeit ausgedrückt, niemals größer sind als die prozentualen Schwankungen in der Höhe der Fließgrenze. Weitere programmäßige Versuche zur Überprüfung des vorliegenden Berechnungsverfahrens der Tragfähigkeit von Stahlbalken wären natürlich außerordentlich erwünscht, wobei, um die Zulässigkeit der Berechnungsgrundlagen genau zu erkennen, angestrebt werden müßte, einen Werkstoff von tunlichst gleichmäßiger Fließgrenze zu verwenden" [15, S. 893]. Damit erkannte Fritsche klar, daß die Fließgrenze des Baustahls die entscheidende Werkstoffkenngröße des Traglastverfahrens darstellt.

\subsection{Karl Girkmann}

Über seine Motive zur Erforschung des elasto-plastischen Verhaltens stählerner Rahmentragwerke schrieb Girkmann: „In meiner Abhandlung ,Bemessung von Rahmentragwerken unter Zugrundelegung eines ideal-plastischen Stahles (s. [16] - d. Verf.) habe ich den Festigkeitsfall Biegung mit Normalkraft behandelt, die Vorgänge während des Belastungsverlaufes in ebenen, rahmenartigen Stabwerken verfolgt und daraufhin den Versuch unternommen, die ,Selbsthilfe' des Stahles bei der Bemessung solcher Tragwerke zu verwerten, um auf diesem Wege zu wirtschaftlicheren Abmessungen solcher Tragwerke zu gelangen. Abgesehen von erzielbaren Gewichtsersparnissen, wäre es bei Anwendung dieses Verfahrens möglich, die Momentengrößtwerte abzuschwächen, die Unterschiede in den erforderlichen Querschnittstärken auszugleichen und damit die konstrulktive Durchbildung zu vereinfachen und zu verbilligen. Sofern die unter den Gebrauchlasten auftretenden Durchbiegungen nicht besonders bestimmt werden müssen, wäre für die rahmenartigen Stabwerke des Hochbaues auch deren Berechnung als statisch unbestimmte Systeme entbehrlich, wodurch sich besonders für die hochunbestimmten Stockwerkrahmen eine ganz wesentliche Vereinfachung der Entwurfsarbeit ergeben würde“ [17, S. 121].

Girkmann entwickelt ein Verfahren der sukzessiven Laststeigerung mit Interaktion, die heute Basis fast aller rechnergestuitzter Berechnungsverfahren ist [18, S. 23-27]. Er verifiziert das von ihm entwickelte Verfahren von statisch unbestimmten Rahmentragwerken an einem zweigelenkig gelagerten Versuchsrahmen durch Dehnungsmessungen. In Bild 6a ist der Versuchsrahmen mit Zugband (oben) und Riegel (unten) dargestellt; er besitzt folgende Parameter:

- Material: Handelsbaustahl mit der Proportionalitätsgrenzc $1,8 \mathrm{t} / \mathrm{cm}^{2}$, den Fließgrenzen $2,58 \mathrm{t} / \mathrm{cm}^{2}$ bzw. $2,67 \mathrm{t} / \mathrm{cm}^{2}$ und den Zugfestigkeiten 4,32 t/c $\mathrm{cm}^{2}$ bzw. 4,27 t/cm

- Ricgellänge (Systemmaß): $1500 \mathrm{~mm}$

- Riegelquerschnitt: 2 U-Profile $80 \mathrm{~mm}$

Ślicllänge (Systemmaß): $595 \mathrm{~mm}$

Slicleuerschnitte: 2 U-Profile $80 \mathrm{~mm}$

Zuy!hanclquerschnitt: 2 Winkelprofile $30 \times 45 \times 4 \mathrm{~mm}$
Sämtliche Walzprofile wurden im Bereich der biegesteifen Rahmenecken, im unteren Stielbereich und im Anschluß Stiel-Zugband durch Bleche kraftschlüssig vernietet.

Bild $6 \mathrm{~b}$ zeigt den Zweigelenkrahmen unter der größten Last; die Meßinstrumente sind bereits abgenommen. Nach der Ehtlastung stellte sich eine scharfe Krümmung in der Riegelmitte ein (Bild 6c), die auf eine gelenkartige Wirkung dieses Bereichs hinweisen (Fließgelenk). In Bild 7 sind die Spannungsbilder der Riegelmitte angegeben. Der kritische Spannungszustand nach Bild 7d ist gekennzeichnet durch die verschwindend klein gewordene Höhe des elastisch gebliebenen Kerns; hierfür leitet Girkmann aus den Gleichgewichtsbedingungen die Plastizitätsbedingung, für die Normalkraft und das Biegemoment ab. Bei einer weiteren Last-
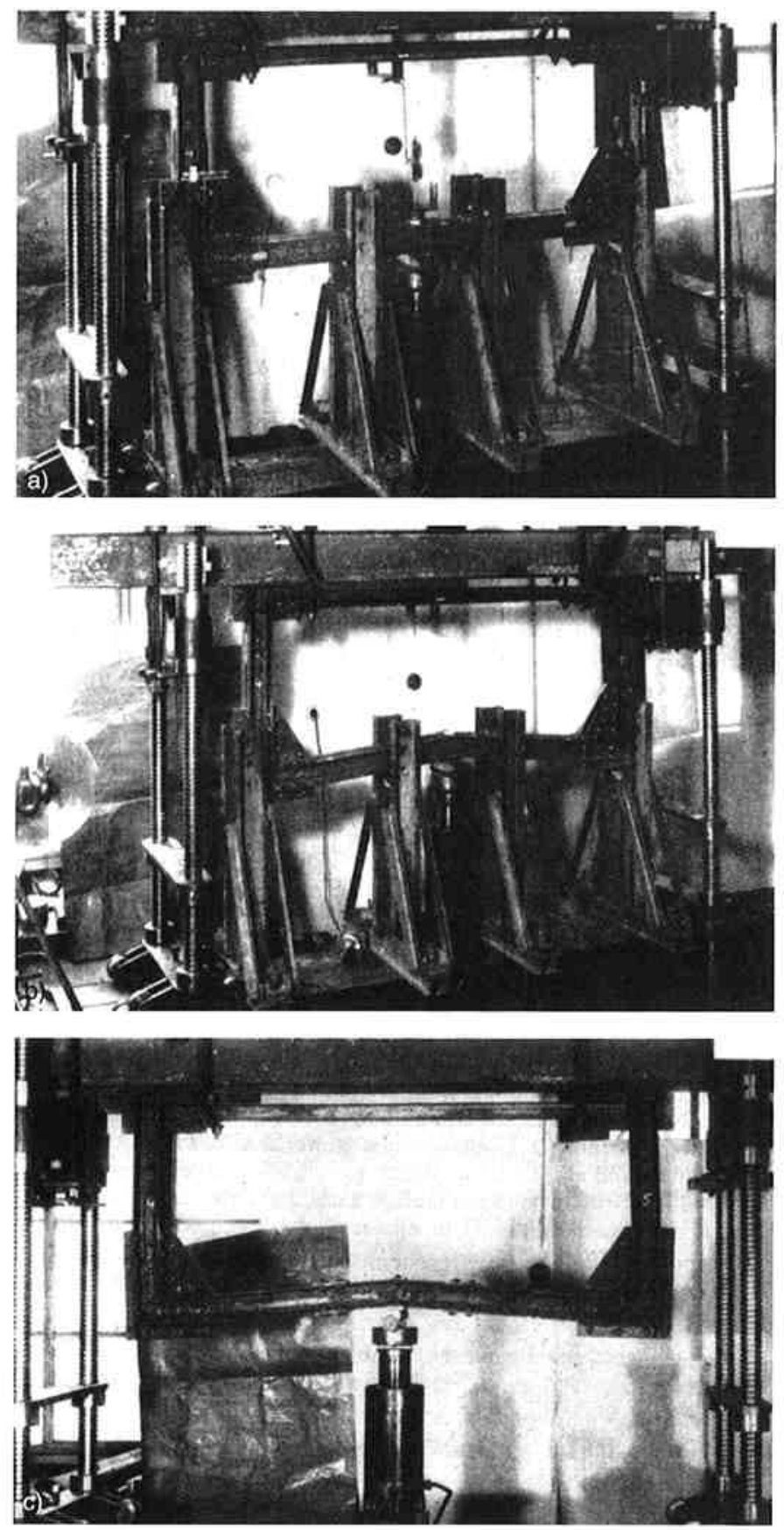

Bild 6. a) Versuchsrahmen; b) Versuchsrahmen unter größter Last (b) und nach Entlastung (c) (Quelle: [17, S. 124/25])

Fig. 6. a) Test frame; test frame under maximal load (b) and after relieving (c) (source: [17, p. 124/25]) 

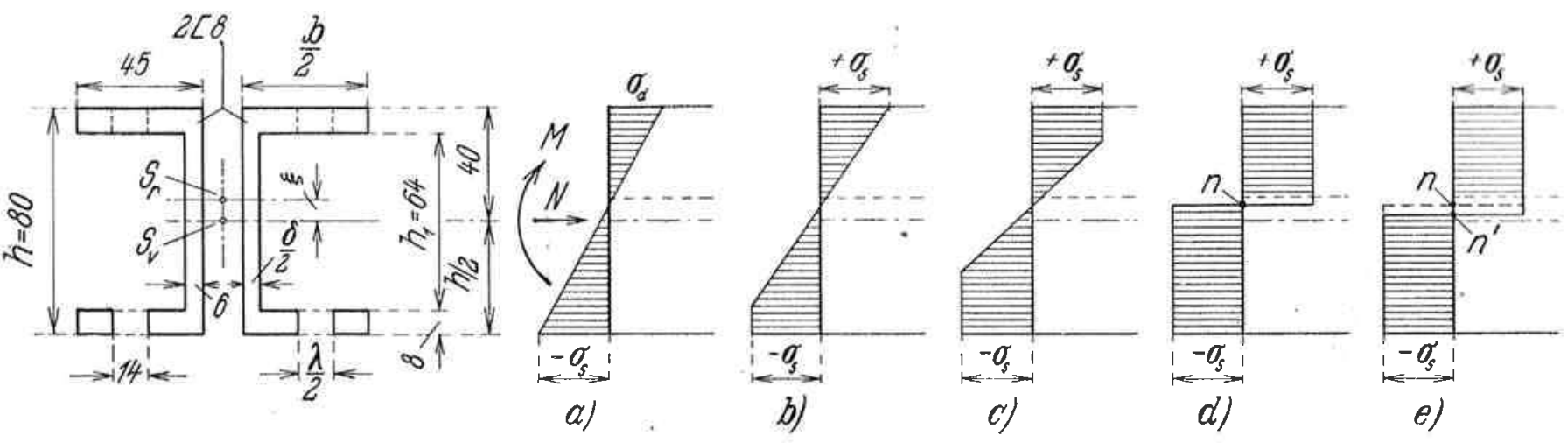

Bild 7. Querschnitt und Spannungsverteilung in Riegelmitte; a) elastischer Grenzzustand; b) und c) teilplastizierter Querschnitt; d) und e) vollplastizierter Querschnitt (Quelle: [17, S. 125])

Fig. 7. Cross section and stress distribution in the middle of the frame; a) elastic limit state; b) and c) partially plastic cross section; d) and e) fully plastic cross section (source: [17, p. 125])

steigerung verschiebt sich lediglich die Null-Linie von $n$ (Bild 7d) nach n' (Bild 7e).

Während bis zur Last nach Bild 7d der Versuchsrahmen als einfach statisch unbestimmtes System aus den elastisch-plastischen Verformungen analysiert werden kann, wirkt er bei weiterer Laststeigerung nach Bild 7e als Dreigelenkrahmen. Bei einem kritischen Lastwert von $\mathrm{P}=$ $\mathrm{P}^{\mathrm{T}}=10,11 \mathrm{t}$ entstehen schließlich Fließgelenke in den Querschnitten O, E und F. (Bild 8). Nach Girkmann stellt die Last $P=P^{T}=10,11 \mathrm{t}$ die nach seinem Verfahren der sulkzessiven Laststeigerung ermittelte Traglast des Versuchsrahmens (Bild 6) dar. Unter dieser Traglast könnten schon beliebig viele Querschnitte Gelenkwirkung erlangen - was hier auch für die Stielquerschnitte $\mathrm{G}$ und $\mathrm{H}$ tatsächlich zutrifft (s. Bild 8).

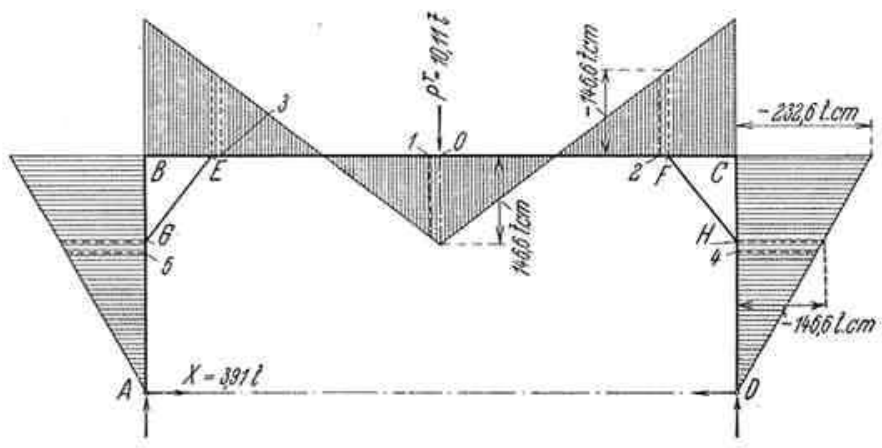

Bild 8. Berechnete Traglast des Versuchsrahmens (Quelle: [17, S, 126])

Fig. 8. Calculated limit load of the test frame (source: [17, p. 126])

Rothert und Gebbeken konnten überzeugend nachweisen, daß Girkmann in seiner Veröffentlichung in der Zeitschrift „Der Stahlbau“ zahlreiche Erkenntnisse zum Traglastverfahren präsentierte, die viele Jahre später von der Forschung mühsam (wieder)errungen werden mußten ([18, S. 26-27]):

1. Wandern der Fließgelenke - exzentrisches Fließgelenk: „Ein Querschnitt mit kritischem Spannungsverlauf wirkt wie ein Gelenk, dessen Lage sich gegenüber der Stabachse stetig ändert" [17, S. 122].

2. Forderung nach positiver Dissipationsarbeit: „Die Wirkung derartiger Gelenke ist immer nur eine beschränkte, denn es sind nur solche gegenseitigen Verdrehungen (..) möglich, bei welchen dieselben im gedrückten Teile der Querschnitte gegeneinander, im gezogenen aber voneinander bewegt werden. Entgegengerichteten Verdrehungen (welche bei Entlastungen zustande kommen) leistet der Baustoff Widerstand, und die Gelenkwirkung schaltet sich in solchen Fällen (...) wieder aus" [17, S. 122].

3. Theorie II. Ordnung: „Das dargelegte Bemessungsverfahren kann auf beliebig gestaltete und belastete Tragwerke angewendet werden, sofern die zusätzlichen Biegungen, welche bei der Verformung der Tragwerke entstehen jeweils vernachlässigbar sind" [17, S. 123].

4. Maßgebliche Schnittgrößen: „Beim Einlegen der Momentenlinien ist noch zu beachten, daß die tatsächlichen Endquerschnitte der Stäbe nicht in die Systemknoten fallen (...), sondern, je nach Ausführungsart der Rahmenecken, in verschieden großen Abständen von den Knoten liegen" [17, S. 123].

5. Órtliches Versagen - Rotationskapazität: „Die zugrunde gelegte tragbare Belastung kann jeweils nur erreicht werden, wenn auch das vorzeitige Falten von Querschnittsteilen (...) der Stäbe vermieden bleibt" $[17$, S. 123].

6. Stabilitätsnachweise: „Die Stabilitätsgrenze des Tragwerks entspricht dem Erreichen der kinematischen Kette. Zusätzlich muß das plastische Kippen einzelner Stäbe vermieden werden. Druckkräfte sollten im Verhältnis der Sicherheitskoeffizienten für Biegung und Knickung erhöht, mit der Knickzahl multipliziert und dann in die Plastizitätsbedingung eingeführt werden" $[18$, S. 27], (s. a. [17, S. 123]).

7. Sicherheitskonzept und Gebrauchsfähigkeit: Es ist zu fordern, „daß unter den Gebrauchslasten selbst bloß elastische Formänderungen zustande kommen (...). Kann hierbei mit der Hebung der Elastizitätsgrenze gerechnet werden (...), dann dürfen die Randspannungen bis zur Streckgrenze ansteigen und es ist bloß zu vermeiden, daß schon unter den Gebrauchslasten die Streckspannung in Querschnitte eindringt. Dieser Bedingung wird (...) immer dann entsprochen, wenn die Festlegung der tragbaren Lasten unter Zugrundelegung einer mindestens zweifachen Sicherheit erfolgt" [17, S. 123].

8. Sicherheit bei wiederholter Belastung: „Aufgrund der Untersuchungen von Fritsche (...) wird der gleiche Sicherheitsgrad gefordert wie für einmalige Belastung" [18, S. 27| (s. a. [17, S. 123/124]). 
9. Bemessung von Anschlüssen: „Stabanschlïsse, Eckverbindungen, Verankerungen usw. sind ebenfalls nach den aus dem zugrunde gelegten Momentenverlauf sich ergebenden Momenten und Kräften so $\mathrm{zu}$ bemessen, daß ein Versagen vor Erreichen der geforderten tragbaren Last vermieden bleibt" [17, S. 123].

Damit lag schon 1932 ein umfassend formuliertes plastisches Bemessungsverfahren für stählerne Stabtragwerke vor.

\subsection{Andere Autoren}

1932 befaßte sich Felix Kann mit der Schnittkraftverteilung von Durchlaufträgern im elastisch-plastischen Bereich; wie Girkmann geht er von der richtigen Spannungsverteilung teilplastizierter Querschnitte von doppelsymmetrischen Stahlprofilen aus [19, S. 106]. 1931 meldete sich in der Zeitschrift „Der Stahlbau“ $v$. Kazinczy zu Wort und notierte die denkwürdigen Sätze: „Gewiß ist dem Konstrukteur auch früher die Spannungs-Dehnungs-Kurve bekannt gewesen, nur hatte er Furcht vor den mathematischen Schwierigkeiten bei Verwendung, obgleich in manchen Fällen auch das Rechnen mit dem Hookeschen Gesetz komplizierter ist. Und jetzt sehen wir, daß die Rechnung nicht verwickelter, sondern einfacher wird. Die wahre Dehnungskurve wurde bis jetzt nur bei dem Problem des Knickens von Kármán verwendet, und die Versuche von Rǒs und Brunner haben es glänzend bestätigt. Also verwenden wir nur tapfer auch in anderen Problemen die Plastizitätstheorie" [20, S. 59]. Dies tat v. Kazinczy wie später gezeigt wird - aber leider ohne durchschlagende Wirkung.

\section{Das Paradoxon des Fließgelenkverfahrens}

So machte Gábor v. Kazinczy schon im Jahre 1931 auf einen Widerspruch im Fließgelenkverfahren aufmerksam [18, S. 29], [21] mit dem 1935 Fritz Stüssi und Curt Fritz Kollbrunner die Fachwelt in Atem hielten [22]: es handelt sich um das Paradoxon des Fließgelenkverfahrens (Bild 9).

Die Last P des Durchlaufträgers wird von Null bis auf den elastischen Grenzzustand gesteigert, so daß die Randfasern an der Stelle des betragsmäßig maximalen Biegemomentes zu fließen beginnen; nach weiterer Laststeigerung entstehen Fließgelenke. Der Grenzzustand der Tragfähigkeit (plastischer Grenzzustand) des Durchlaufträgers ist dann erreicht, wenn sich in den Querschnitten (1), (2) und (3) Fließgelenke ausbilden: Durch diese drei Fließgelenke hat sich das zweifach statisch unbestimmte System in ein einfach kinematisch bestimmtes System verwandelt. Die zu dieser Fließgelenkkette (kinematischen Kette) gehörende Last wird Traglast T oder plastische Grenzlast T des Durchlaufträgers genannt (Bild 9/rechts).

Beim Einfeldträger dagegen (Bild 9/links) kann sich bei Laststeigerung nur im Querschnitt (2) ein Fließgelenk einstellen. Wird die Traglast der beiden in Bild 9 dargestellten Systeme verglichen, dann beträgt die Traglast des Durchlaufträgers das Doppelte der Traglast des Einfeldträgers. Stüssi und Kollbrunner betrachten nun zwei Grenzfiille. Geht $\ell_{1} \rightarrow 0$, dann verwandelt sich der DurchlaufträBer in Bild 9/rechts in einen beidseitig eingespannten Trä- ger der Spannweite $\ell$ mit der Traglast $\mathrm{T}=8 \mathrm{M}_{\mathrm{p} \ell} / \ell$; diese Traglast stimmt mit der des Durchlaufträgers überein. Wird dagegen der Grenzübergang $\ell_{1} \rightarrow \infty$ vollzogen, dann bildet sich der Durchlaufträger um in einen frei aufliegenden Einfeldträger der Spannweite $\ell$ mit derTraglast $\mathrm{T}=4 \mathrm{M}_{\mathrm{p} \ell} / \ell$; diese Traglast beträgt nur die Hälfte der Traglast des Durchilaufträgers. Damit ist das Paradoxon der Fließgelenktheorie benannt. Stüssi und Kollbrunner resümieren: „Das Traglastverfahren gibt somit gegenüber einfachen Balken zu große Werte für die Tragfähigkeit, verzichtet also auf eine gewisse innere Tragwerkreserve (Verfestigungsbereich). Nach der Elastizitätstheorie bemessene statisch unbestimmte Tragwerke besitzen gegenüber statisch bestimmten einen Sicherheitsüberschụß" $[22, \mathrm{~S}$. 267]. Damit plädierten beide Autoren für die Rückkehr zur Elastizitätstheorie, da das Traglastverfahren in dem von ihnen untersuchten Durchlaufträger auf der unsicheren Seite liegt; ihre Devise ist: „Sicherheit - sich absichern" [23].

Rothert und Gebbeken entdeckten, daß v. Kazinczy schon 1931 das Paradoxon des Fließgelenkverfahren klar erkannte und sogar schon darauf hinwies, daß die Durchbiegung $\mathrm{f}_{2}$ des Durchlaufträgers beim Grenzübergang $\ell_{1} \rightarrow \infty$ eine unerlaubte Größe erlangen, wie sich $v$. Kazin$c z y$ ausdrückte [18, S. 29]. Leider wurde diese Veröffentlichung damals nicht rezipiert.

Obwohl Maier-Leibnitz den Stüssi-Balken (Bild 9/ rechts) ein Jahr später im Versuch nachfuhr [24] und Fritsche aus der Perspektive der Plastizitätstheorie das Paradoxon der Fließgelenktheorie reflektierte [25], gelang ihnen keine überzeugende Klärung. Die Elastizitätstheorie als Bemessungsgrundlage für Stahlbauten triumphierte in den deutschsprachigen Ländern über die Plastizitätstheorie als Grundlage des Fließgelenkverfahrens.

Unter der Federführung Stüssis wurde auf der Berliner Olympiade der Konstrultiven Bauingenieure 1936, dem zweiten Kongreß der Internationalen Vereinigung für Brückenbau und Hochbau (IVBH), massive Kritik am Fließgelenkverfahren geübt; dazu schrieb Stüssi rückblickend: „Im Anschluß an die Diskussionen und Gespräche am Berliner Kongreß 1936 der Internationalen Vereinigung für Brückenbau und Hochbau wurde es allerdings sehr rasch still um diese Bemühungen (Fließgelenkverfahren als Bemessungsgrundlage für Stahlbauten - d. Verf.) und die zuständigen Fachleute des Stahlbaues waren sich mehr und mehr einig darüber, daß die Einführung des Traglastverfahrens eine Qualitätsverschlechterung der Bauweise bedeuten würde“ [26, S. 53]. Erst 1952 klärten Symonds und Neal das Paradoxon der Traglasttheorie auf [27]. Sie untersuchten die Durchbiegungen, die relativen Drehungen und die Dehnungen des Fließgelenks und fanden, daß insbesondere die Rotation des Fließgelenks von einer bestimmten Spannweite der Endfelder $\ell_{1}$ an unzulässig groß werden; im Grenzfall $\ell_{1} \rightarrow \infty$ geht auch die Durchbiegung des Punktes (2) (Bild $9 /$ rechts) $f_{2} \rightarrow \infty$, so daß die Rechnung ihren Sinn verliert und nicht als Grenzfall des frei aufliegenden Einfeldträgers aufgefaßt werden darf [28, S. 152].

Wer dem Paradoxon gegenübersteht, setzt sich der Wirklichkeit aus, läßt der Schweizer Schriftsteller Friedrich Dürrenmatt die Physiker in seinem Stück „Die Physiker" sagen. 
Problem:

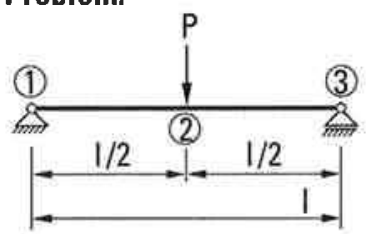

\section{Stoffgesetz:}
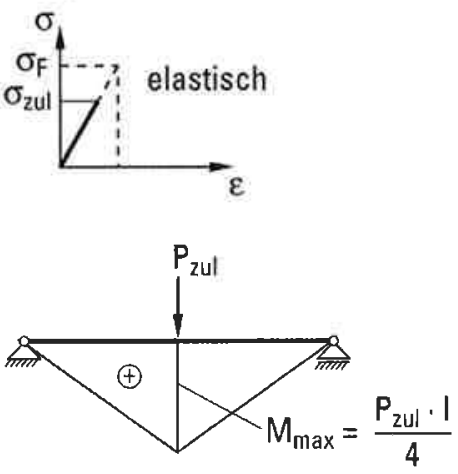

Punkt(2):

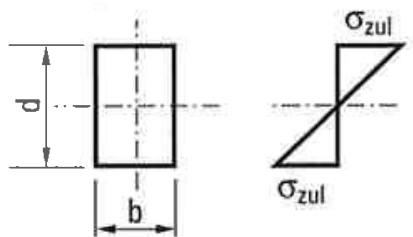

Bemessung:

$M_{\text {max }}=M_{\text {zul }}=\sigma_{\text {zul }} \cdot \frac{b \cdot d^{2}}{\frac{6}{W}}$

Nun wird die Last zur Ausbildung eines Fließgelenkes in (2) gesteigert $M_{p l_{(2)}}=\sigma_{F} \cdot \frac{b \cdot d^{2}}{4}$

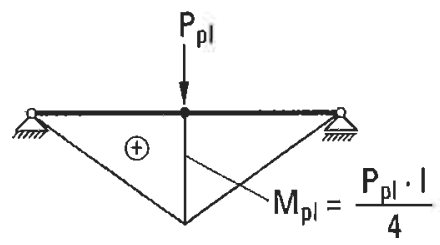

$\longrightarrow P_{p l}=\frac{4 M_{p l}}{l}=T$

$$
2 P_{p l}=P_{p l}^{\prime}
$$

\section{entspricht der Traglast T}

$$
\mathrm{T}=\mathrm{P}_{\mathrm{pl}}\left\langle\text { } \text { Widerspruch } \lesssim \mathrm{T}^{\prime}=2 \mathrm{P}_{\mathrm{pl}}\right.
$$
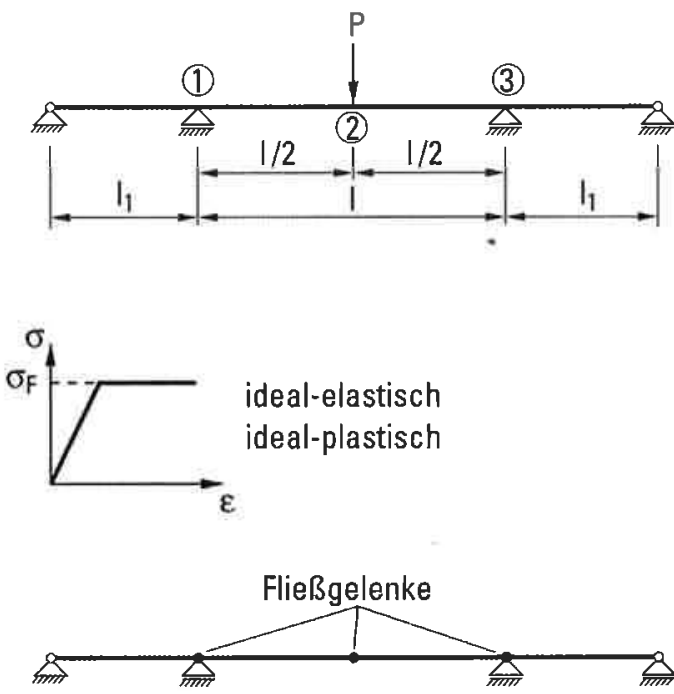

dieser Zustand wird in 3 Schritten erreicht:

1) ideal-elastisch $\sigma \leq \sigma_{\mathrm{F}}$

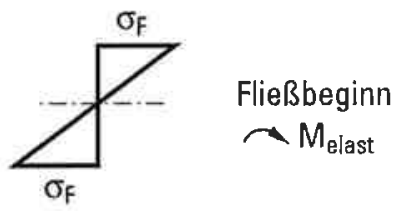

2) teilweise plastiziert
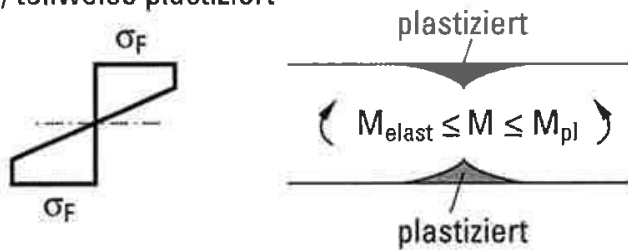

3) vollplastiziert (Fließgelenk)

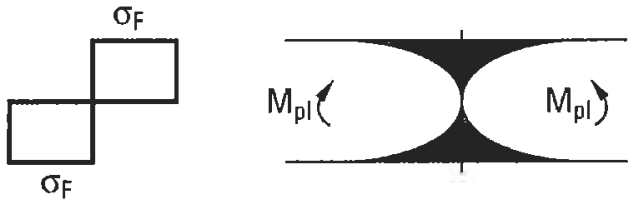

$M_{p l_{(1)}}=M_{p l_{(2)}}=M_{p l_{(3)}}=\sigma_{F} \cdot \frac{b \cdot d^{2}}{4}$

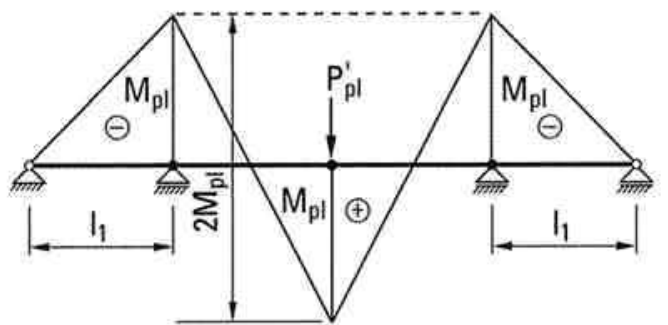

$\longrightarrow 2 \mathrm{M}_{\mathrm{pl}}=\frac{\mathrm{P}_{\mathrm{pl}}^{\mathrm{i}} \cdot \mathrm{l}}{4} \longrightarrow \mathrm{P}_{\mathrm{pl}}^{\prime}=\frac{8 \mathrm{M}_{\mathrm{pl}}}{\mathrm{I}}=\mathrm{T}$

für $\mathrm{l}_{1} \rightarrow \infty$ geht aber obiger Durchlaufträger in einen Träger auf 2 Stützen über (s. links o.)

Bild 9. Paradoxon der Fließgelenktheorie (Quelle: [5, S. 81]) Fig. 9. Paradox of plastic-hinge method (source: $[5, p .81]$ ) 


\section{Durchsetzung des Traglastverfahrens}

Fragt man heute nach der führenden Stellung des Stahlbaus im Bauwesen Großbritanniens, dann wird klar, daß ein wichtiges Moment die frühe und breite Einführung des Traglastverfahrens zur Bemessung von Stahlhochbauten in Großbritannien darstellt, die mit den Namen John Fleetwood Baker und Jacques Heyman verbunden ist.

\subsection{John Fleetwood Baker}

1929 begann auf Anregung des britischen Stahlbau-Verbandes (British Steelwork Association) der Ausschuß für Stahlbauforschung (Steel Structures Research Committee SSRC) unter Leitung von John F. Baker mit seiner Arbeit, den Entwurf, die Berechnung und Konstruktion von Stahlhochbauten zu bilanzieren mit dem Ziel der Neuordnung des britischen Stahlhochbaus. Durch Bakers Arbeit erreichte das triadische Zusammenspiel von Stahlbauindustrie, Stahlbauwissenschaft und Stahlbauvorschriften ein Niveau, das eine neue Sicht auf das Tragverhalten von Stahllkonstrulktionen schon früh erlaubte. Die von ihm angeregten Versuche sowie die kritische Reflektion der auf der Elastizitätstheorie basierenden Bemessung von Stahlskelettbauten und deren Umsetzung im Vorschriftenwerk legte das SSCR 1931, 1934 und 1936 in umfangreichen Berichten nieder [8.73, S. 136]. Auf dem zweiten Kongreß der Internationalen Vereinigung für Brückenbau und Hochbau (IVBH) in Berlin 1936 bilanzierte Baker mit seinem Beitrag „A new method for the design of steel building frames“ [29] seine Tätigkeit als Technical Officer der SSRC auf der Ebene der Bemessungstheorie. Dort lernte er auch Maier-Leibnitz' Traglastversuche kennen, was ihn veranlaßte, weitere Versuchsprogramme zum plastischen Verhalten von Stahlkonstruktionen zu initiieren mit dem Ergebnis, daß schon 1948 der British Standard 449 ,The use of structural steel in building" in einem Absatz die Bemessung von Stahlkonstruktionen nach dem Traglastverfahren zuließ. 1949 publizierte Baker ein Probierverfahren zur Ermittlung der Biegemomentenverteilung in stählernen Rahmen im plastischen Grenzzustand [30]; auf die Arbeiten Girkmanns aus den Jahren 1931/32 ging er dabei nicht ein [8, S. 49-50].

\subsection{Exkurs: Ein Rechenbeispiel}

Bild 10 zeigt die Berechnung eines einseitig eingespannten Einfeldträgers nach der statischen (Bild 10/links) und nach der kinematischen (Bild 10/rechts) Methode. Die kinematische Methode für Rahmentragwerke entwickelten Symonds und Neal 1950/51 [31].

Das Verfahren I (Bild 10/links) geht von der Annahme eines plausiblen, zulässigen Kraftzustandes zur gegebenen Belastung aus; es wird deshalb statische Methode genannt. Daran schließt sich die Berechnung des Laststeigerungsfaktors $v$ an, und zwar unter Beachtung der Plastizitätsbedingung $\mathrm{M}=\mathrm{M}_{\mathrm{p} \ell}$ in den Fließgelenken und $\mathrm{M}$ kleiner $\mathrm{M}_{\mathrm{p} \ell}$ in den übrigen Bereichen. Schließlich wird der Verschiebungszustand auf geometrische Verträglichkeit geprüft (kinemalische Kontrolle): im plastischen Grenzzustand muß sich cine verträgliche (einfach kinematisch bestimmte) Fließmolonkkelte ergeben
Die kinematische Methode (Verfahren II im Bild 10/ rechts) beginnt mit der Annahme von plausiblen, zulässigen Fließgelenkketten. Mit dem Prinzip der virtuellen Verschiebungen, d. h. der Gleichheit der äußeren virtuellen und der negativen inneren virtuellen Arbeit werden im zweiten Schritt die Laststeigerungsfaktoren berechnet. Da sowohl die äußere als auch die innere virtuelle Arbeit für sich immer minimal sein müssen, ist jene Fließgelenklkette maßgebend, aus der sich der kleinste Laststeigerungsfaktor $v_{\min }$ ergibt. Abschließend muß geprüft werden, ob die Plastizitätsbedingung $\mathrm{M} \leq \mathrm{M}_{\mathrm{p} \ell}$ erfüllt ist.

\subsection{Kontroverse um das Traglastverfahren}

Auf der zweiten Schweizerischen Stahlbau-Tagung 1956 wiederholte der 1937 zum Professor für Baustatik, Hochund Brückenbau in Stahl und Holz an die ETH Zürich berufene Fritz Stüssi seine mit dem Paradoxon der Fließgelenktheorie begriindete Kritik und berief sich $\mathbf{u}$. a. auf Autoritäten wie Leonardo da Vinci und Navier [18, S. 34]. Dessen ungeachtet stellte Bruno Thürlimann auf der Grundlage von Versuchen das Fließgelenkverfahren in der Schweizerischen Bauzeitung vor [32]. Diese Versuche wurden an der Lehigh University, Bethlehem - einem führenden Zentrum der Stahlbauforschung in den USA - durchführt und ausgewertet; dort promovierte sich Thürlimann und wirkte von 1953 bis 1960 als Professor, ehe er 1960 an die ETH Zürich zum Professor für Baustatik, Hoch- und Brückenbau in Stein, Beton und vorgespanntem Beton ernannt wurde ${ }^{2}$. In seinem umfassenden, klar aufgebauten Beitrag untersucht Thürlimann statische Systeme, an denen er die Unzulänglichkeit der elastischen Berechnungsmethoden aufweist (Paradoxon der Elastizitätstheorie); ferner analysiert er den Stüssi-Balken. Thürlimann gibt eine allgemeine, auf dem Prinzip der virtuellen Verschiebungen beruhende Darstellung der kinematischen Methode des Fließgelenkverfahrens (s. a. Bild 10/rechts). Hierauf reagierte Stüssi allergisch. Zum einen verweist er auf das Paradoxon der Fließgelenktheorie, zum anderen bestreitet er den Momentenausgleich durch die Bildung von Fließgelenken und die von ihm gefundene Zulässigkeit der Anwendung des Prinzips der virtuellen Verschiebungen: „Ein statisch unbestimmtes Tragwerk bleibt auch dann statisch unbestimmt, wenn in einzelnen Querschnitten die Proportionalitätsgrenze oder die Fließgrenze des Materials überschritten wird. Dies bedeutet, $\mathrm{da} ß$ auch im überelastischen Belastungsbereich neben den Gleichgewichtsbedingungen auch die Formänderungsbedingungen gültig bleiben. Das Ungenügen des Traglastverfahrens beruht darauf, daß es diese grundlegende Tatsache mißachtet, und seine ,Einfachheit' erweist sich bei näherer Prüfung als unzulässige Primitivität (...). Sollte jedoch die Befürwortung des Traglastverfahren als Entgegenkommen an jene Leute gedacht sein, die die normalen und bei normalen Begabungen erlernbaren Methoden zur Berechnung statisch unbestimmter Tragwerke nicht beherrschen, so müßte die Einführung einer solchen ,Baustatik für Schwachbegabte' erst recht abgelehnt werden“" [26, S. 57].

Dem Elastiker Stüssi ist der Gedanke der Störung des elastischen Kontinuums durch Fließgelenke und damit die

2 Nach der Emeritierung Stüssis wurde Thürlimanns Professur 1973 in „Baustatik und Konstruktion“ umbenannt. 
$\mathrm{P}=200 \mathrm{kN}$

$\mathrm{M}_{\mathrm{pl}}=500 \mathrm{kNm}$

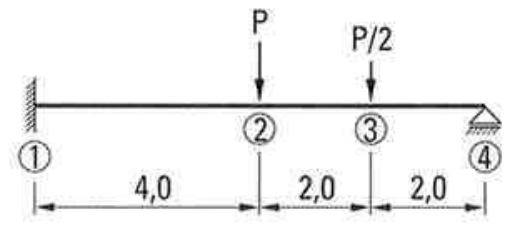

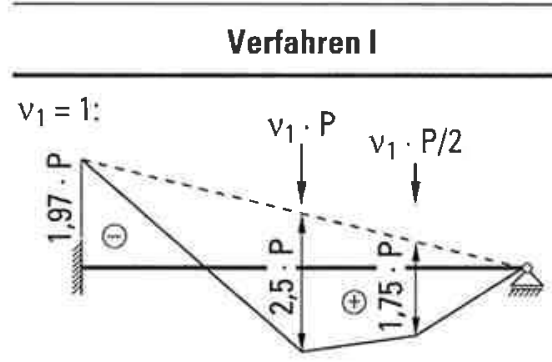

$M_{(1)}^{(1)}=-1,97 P=394<M_{p l}$

$M_{(2)}^{(1)}=(2,5-1,97 / 2) P=1,515 P=303<M_{p l}$

$M_{(3)}^{(1)}=(1,75-1,97 / 4) P=1,258 P=255<M_{p l}$

$\longrightarrow$ bei $v_{1}=1$ plastiziert der Träger nicht

$-v_{2}=\frac{500}{394}=1,27$

bei $v_{2}=1,27$ plastiziert die Stelle (1)

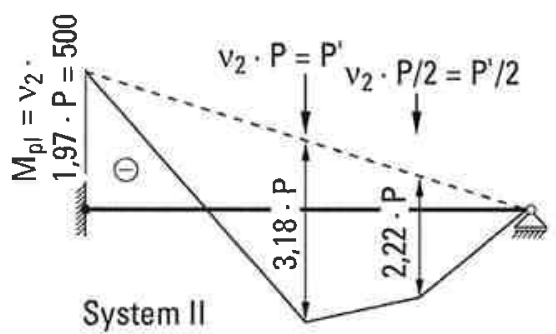

$\rightarrow$ das statische System hat sich geändert, da $M_{(1)}^{(1)}=M_{p l}$ nicht mehr gesteigert werden kann

$\mathrm{M}_{(2)}^{(0)}=1,515 \mathrm{P} \cdot 1,27=1,92 \mathrm{P}=394<\mathrm{M}_{\mathrm{pl}}$

$M_{(3)}^{(0)}=1,258 P \cdot 1,27=1,6 P=320<M_{p l}$

"neues" statisches System (stat. best.)

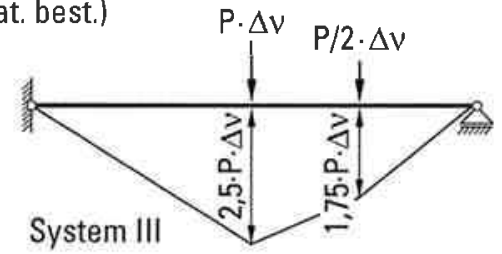

System IV = System II + System III:

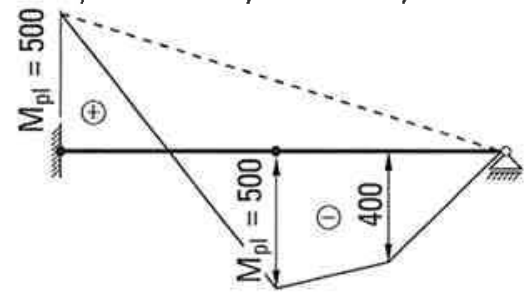

$\longrightarrow$ in Punkt (2) bildet sich das

2. Fließgelenk aus:

$394+2,5 \cdot P \cdot \Delta v=500=M_{p l}$

$\rightarrow \Delta v=0,23$

$\rightarrow v=1,27+0,23=1,5$

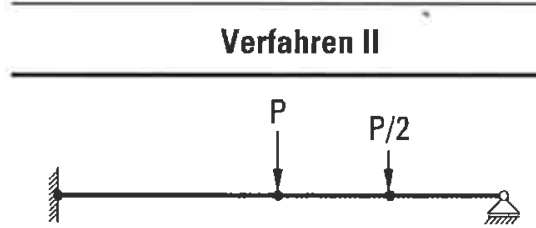

$z=3$ ( 3 mögliche Fließgelenke)

$\rightarrow \mathrm{u}=\mathrm{z}-\mathrm{n}=3-1=2$

$\longrightarrow u_{1}, u_{2}$ und die Kombination $\mathrm{u}_{3}=\mathrm{u}_{1}+2 \mathrm{u}_{2}$

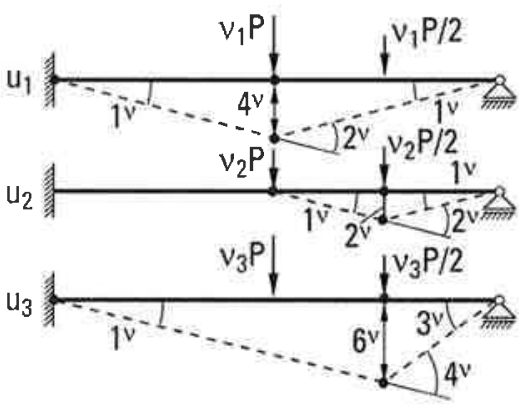

Arbeitsgleichung: $W_{a}{ }^{v}=-W_{i}{ }^{v}$

$$
\begin{aligned}
W_{a}^{v} & =-W_{i}^{v} \\
u_{1}: v_{1}\left(P \cdot 4^{v}+P / 2 \cdot 2^{v}\right) & =M_{p l} \cdot\left(1^{v}+2^{v}\right) \\
-v_{1} & =1,5=v_{\min } \\
u_{2}: v_{2}\left(P \cdot 0^{v}+P / 2 \cdot 2^{v}\right) & =M_{p l} \cdot\left(1^{v}+2^{v}\right) \\
v_{2} & =7,5 \\
u_{3}: v_{3}\left(P \cdot 4^{v}+P / 2 \cdot 6^{v}\right) & =M_{p l} \cdot\left(1^{v}+4 v\right) \\
\longrightarrow v_{3} & =1,785
\end{aligned}
$$

$v_{\min }=1,5 \rightarrow$ maßgebender Faktor bei dem die Traglast erreicht wird

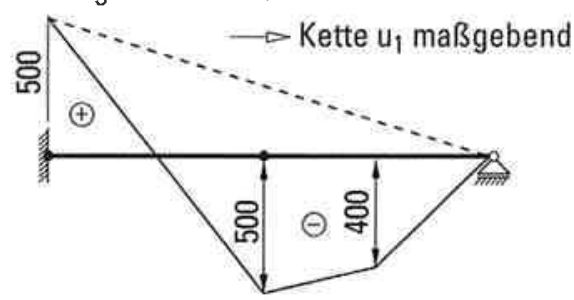

Plastizitätskontrolle: $M \leq M_{p l}$ ist erfüllt!

Vorzeichen

$$
M_{i}=M_{p l} 3-j_{\Delta \varphi}^{-1} M_{i}=M_{p l}
$$

Die inneren Momente $M_{i}=M_{p l}$ wirken immer so, daß sie den Winkelsprung $\Delta \varphi$ $\mathrm{zu}$ beseitigen suchen

$W_{i}=-M \cdot \Delta \varphi \longrightarrow-W_{i}=M \cdot \Delta \varphi$

die negative innere Arbeit ist immer positiv

Die Traglast des Systems ist also

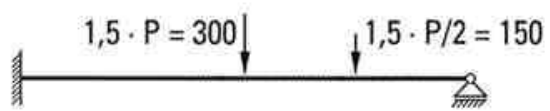

Bild 10. Berechnung eines einseitig eingespannten Einfeldträgers nach der statischen Methode (links) und der kinematischen Methode (rechts) (Quelle: [5, S. 86])

Fig. 10. Calculation of a fixed-ended beam using the static method (left) and the kinematic method (right) (source: $[5, p .86]$ ) 
Negation der Formänderungsbedingungen, aus denen ja die zur Bestimmung der statisch unbestimmten Größen in elastischen Tragwerken erforderlichen Elastizitätsgleichungen abgeleitet werden, überaus fremd. Er beharrt auf der Ausschließlichkeit des Wahrheitwertes des ihm in Fleisch und Blut übergegangenen Selektionmechanismus der Formänderungsbedingungen, mit dem aus der unendlichen Mannigfaltigkeit von möglichen Gleichgewichtszuständen in statisch unbestimmten Systemen der wahre selektierbar ist; dagegen geht die Fließgelenktheorie von wahrscheinlichen Gleichgewichtszuständen aus, deren Wahrheitswert sie beispielsweise über das Prinzip der virtuellen Verschiebungen oder - für Stüssi noch schlimmer - über Probierverfahren überprüft. In der Kontroverse zwischen Stüssi und Thürlimann scheint die alte Differenz auf zwischen der geometrischen Richtung der Statik (Suche nach wahren Gleichgewichtszuständen) und der kinematischen Richtung der Statik (physikalisch begründete Setzung von Bruchmechanismen aus der Menge der möglichen Gleichgewichtszustände) - eine Differenz, die philosophisch in dem erstmals von Aristoteles erkannten logischen und ontologischen Status der Differenz von Möglichkeit und Wirklichkeit begründet liegt.

In seiner Stellungnahme entzaubert Thürlimann nach Symonds und Neal das Paradoxon der Fließgelenktheorie [33]. Doch Stüssi läßt nicht locker und bewertet das Traglastverfahren als unbefriedigendes Näherungsverfahren, läßt aber seine Behauptung, daß es zu unsicheren Rechenergebnissen führt, fallen [8, S. 66]. Gleichwohl hat Stüssis Kritik am Traglastverfahren dazu beigetragen, daß in den 1960er Jahren auf der Plastizitätstheorie fußende baustatische Verfahren entwickelt wurden. So faßte 1963 Charles Massonnet den Streit um das Fließgelenkverfahren auf und definiert in einem Dreieckdiagramm die Anwendungsgebiete der Elastizitäts-, Plastizitäts- und Viskoelastizitätstheorie [34]. Massonnet war es auch, der maßgebend die europäischen Empfehlungen für die plastische Bemessung von Stahltragwerken in den 1970er Jahren vorantrieb [35].

Mit seiner 1964 fertiggestellten und 1965 veröffentlichten Habilitationsschrift ,Die Traglastberechnung stählerner Rahmentragwerke nach der Plastizitätstheorie II. Ordnung" [36] schuf Udo Vogel das Fließgelenkverfahren nach Theorie II. Ordnung. Dort bestimmt Vogel den kritischen Lastfaktor durch ein von ihm gefundenes Iterationsverfahren; es ähnelt jenem Verfahren, das Alfred Teichmann zur Lösung des Stabilitätsproblems nach der Deformationstheorie II. Ordnung vorgeschlagen hatte: Nichtlineare Theorien führen auf nichtlineare Gleichungssysteme, die i. d. R. nur iterativ gelöst werden können. Iterationsverfahren wiederum begünstigten den Einsatz von Computern für statische Berechnungen wie umgekehrt der Computer sich zum Mittel der baustatischen Theoriebildung im nichtlinearen Bereich entwickelte. Diese Wechselwirkung sollte ein Wesensmerkmal der Diffusionsphase der Baustatik (seit 1975) bilden.

\subsection{Jacques Heyman}

1946 trat Jacques Heyman, der 1944 sein Ingenieurstudium ahgeschlossen hatte, Bakers Team bei, wo er bald wichtige Aulgabenbereiche übernehmen konnte. 1949 schloß er seine Promotion ab. Kurz darauf reiste er in die Vereinigten Staaten, um mit Wilhelm (William) Prager zusammenzuarbeiten. Professor Prager war unmittelbar nach der Machtübertragung an die NSDAP aus Deutschland geflohen und 1941 dem renommierten Fachbereich Mathematik an der Brown University beigetreten, wo er sich mit Forschungsarbeitèn zum Thema Plastizität befaßte. Baker und Prager bauten ein Austauschprogramm zwischen Brown und Cambridge auf. Diese Zusammenarbeit sollte sich als entscheidend erweisen. Prager hatte aufgezeigt, daß sich die drei Aussagen zur Mechanik von Festkörpern (Gleichgewichts- und Elastizitätsgleichungen sowie Materialeigenschaften) in der klassischen Elastizitätstheorie in einer einzigen Gleichung vereinen lassen, während sich in der Plastizitätstheorie ein völlig anderes Bild ergibt [37]. Dieser neue Lösungsansatz sollte sich für die spätere strengere Formalisierung der Plastizitätstheorie für Stahlskelette als grundlegend erweisen. Austauschaufenthalte für promovierte wissenschaftliche Mitarbeiter waren in der Regel für ein Jahr vorgesehen. Jacques Heyman blieb jedoch drei Jahre lang an der Brown University, und bei seiner Rückkehr im Jahr 1952 hatte er umfassende theoretische Grundkenntnisse erworben. 1956 veröffentlichte er zusammen mit J. F. Baker und M. R. Horne das erste grundlegende Buch zur Plastizitätstheorie für Stahlskelette: „The steel skeleton. Vol. 2: Plastic behaviour and design" [2], [8.19]. Das Buch faßte sämtliche Arbeiten des Teams aus Cambridge aus den vergangenen 10 Jahren zusammen, und es war das erste Buch aus dem Bereich der technischen Analytik, das die grundlegenden Theoreme der Traglasttheorie erwähnte und praktische Anwendungen beschrieb.

Diese grundlegenden Theoreme hatte der sowjetische Ingenieur A. A. Gvozdev 1936 nachgewiesen [38]. Sie waren jedoch Iediglich 1938 auf Russisch in der im Westen weniger bekannten Schriftenreihe der Moskauer Akademie der Wissenschaften veröffentlicht worden und blieben der internationalen Wissenschaftlergemeinschaft daher weitgehend verborgen. Anfang der 1950er Jahre wurden die Theoreme von Pragers Team sozusagen wieder entdeckt (Bild 11). Die Anwendung der Theoreme zur Analyse von Stahlskeletten ermöglichte den konsequenten Einsatz der Berechnungen nach der Plastizitätstheorie, die seit 1948 in Großbritannien durchgeführt worden waren (der relevante Britische Code wurde um eine entsprechende Klausel erweitert). Im Rahmen von Teamarbeit ist es oft schwer, die Verdienste Einzel-

Theorem I.-The a afety factor against collapse is the largest statically admissible multiplier.

Theorem II.-The work that the collapse louds do on tlie dieplacements of their points of application must equal the work that the limil moments in the yicld hinges do on the relutive rotutions of the partg connecled by the hiliges.

Theorem III.-The safely factor aguinst collapse is the smallest kinematically sufficient multiplier.

Theorem IV.-Collapse cannot accur under the louds obtained by multiplying the given loada by a factor which is smaller than a statically admissible multiplier.

Theorem V.-If a beam or frame is strengthened (that is, if its cross sections are changed in auch a manner that the limit moment is increased for, at least, one cross section and decreased for none), the safety factor for a given system of loads cannot decrease as a result of this atrengthening.

Bild 11. Traglast-Theoreme nach Greenberg und Prager (Quelle: [39])

Fig. 11. Theorems of limit load design acc. to Greenberg and Prager (source: [39]) 
ner herauszustellen. Es läßt sich jedoch unschwer erkennen, daß Professor Heyman bei der Ausarbeitung der Plastizitätstheorie für die Anwendung auf Stahlskelette eine zentrale Rolle spielte. Das Literaturverzeichnis am Ende dieses Aufsatzes zeigt, daß er an fast allen Arbeiten zu theoretischen und praktischen Aspekten beteiligt war.

Die plastizitätstheoretisch fundierte Baustatik erlebte zwischen 1960 und 1970 ihre Konsolidierungsphase. Spezialstudien wurden durch die Veröffentlichung von Handbüchern abgelöst, die eine entscheidende Rolle für die Anwendung der Theorie auf breiter Basis spielten. Bei den Verfassern dieser Handbücher handelte es sich um Persönlichkeiten, die wesentlich an diesem Prozeß beteiligt waren, darunter Beedle, Neal und Horne. 1966 veröffentlichte auch Heyman zusammen mit Baker ein Handbuch in dieser Serie, das sich als Modell fürviele spätere Beiträge erwies [8.46].

Die plastizitätstheoretisch fundierte Baustatik wurde zunächst für Stahlkonstruktionen entwickelt. Später erkannte man, daß sie auch auf Stahlbetonkonstruktionen anwendbar war. Wie sich weiter herausstellte, kann die Plastizitätstheorie auf beliebige Konstruktionen mit duktilem Verhalten angewandt werden, sofern keine Stabilitätsprobleme vorliegen. Jacques Heyman legte diese Tatsache, die einige Ingenieure bereits seit Beginn des 20. Jahrhundert vermutet hatten, klar und deutlich dar. Er erkannte als Erster, daß sich aus den grundlegenden Theoremen ein neues Paradigma ergab, das sich auf sämtliche mit konventionellen Materialien errichtete Konstruktionen anwenden ließ. Für Stahlbeton könnte dies unter Umständen of- fensichtlich erscheinen. So befaßte sich z. B. v. Kazinczy 1933 [40] und Gvozdev 1936 [38] mit der Analyse von Traglasten in Stahlbetonkonstruktionen. Für Materialien wie zum Beispiel Holz war die Anwendung jedoch weniger offensichtlich, ganz zu schweigen von Mauerwerk. Jacques Heyman erkannte jedoch, daß die Theoreme der Traglasttheorie ftir heterogene Materialien wie zum Beispiel Naturstein oder Ziegel entsprechend angepaßt werden konnten.

Sein 1966 veröffentlichter Aufsatz „The Stone Skeleton" stellte einen Meilenstein für die Entwicklung der modernen Theorie des Mauerwerkbaus dar. Dieser höchst originelle und einleuchtende Aufsatz erläutert die Anpassung der Plastizitätstheorie auf den Mauerwerksbau. Einem Hinweis Pragers [41] folgend erkannte Heyman, daß die grundlegenden Theoreme auf solche im Grunde inhomogenen Konstruktionen angewandt werden können, wenn man dem „Material Mauerwerk" bestimmte Eigenschaften zuweist. Seinem ersten Artikel aus dem Jahr 1966 folgten mehr als 30 weitere Artikel und diverse Bücher zum Thema Mauerwerk. ${ }^{3}$ In diesen Veröffentlichungen wandte er die moderne Theorie auf die Untersuchung von Grundelementen des Mauerwerks wie z. B. Gewölbe, Kuppeln, Strebebögen und Türme an.

Durch seine theoretischen Überlegungen zur Gotik konnten die seit Mitte des 19. Jahrhunderts andauernden

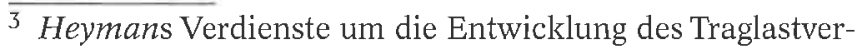
fahrens für Konstruktionen aus Mauerwerk werden demnächst von den Autoren in der Zeitschrift „Mauerwerk“ gewürdigt.
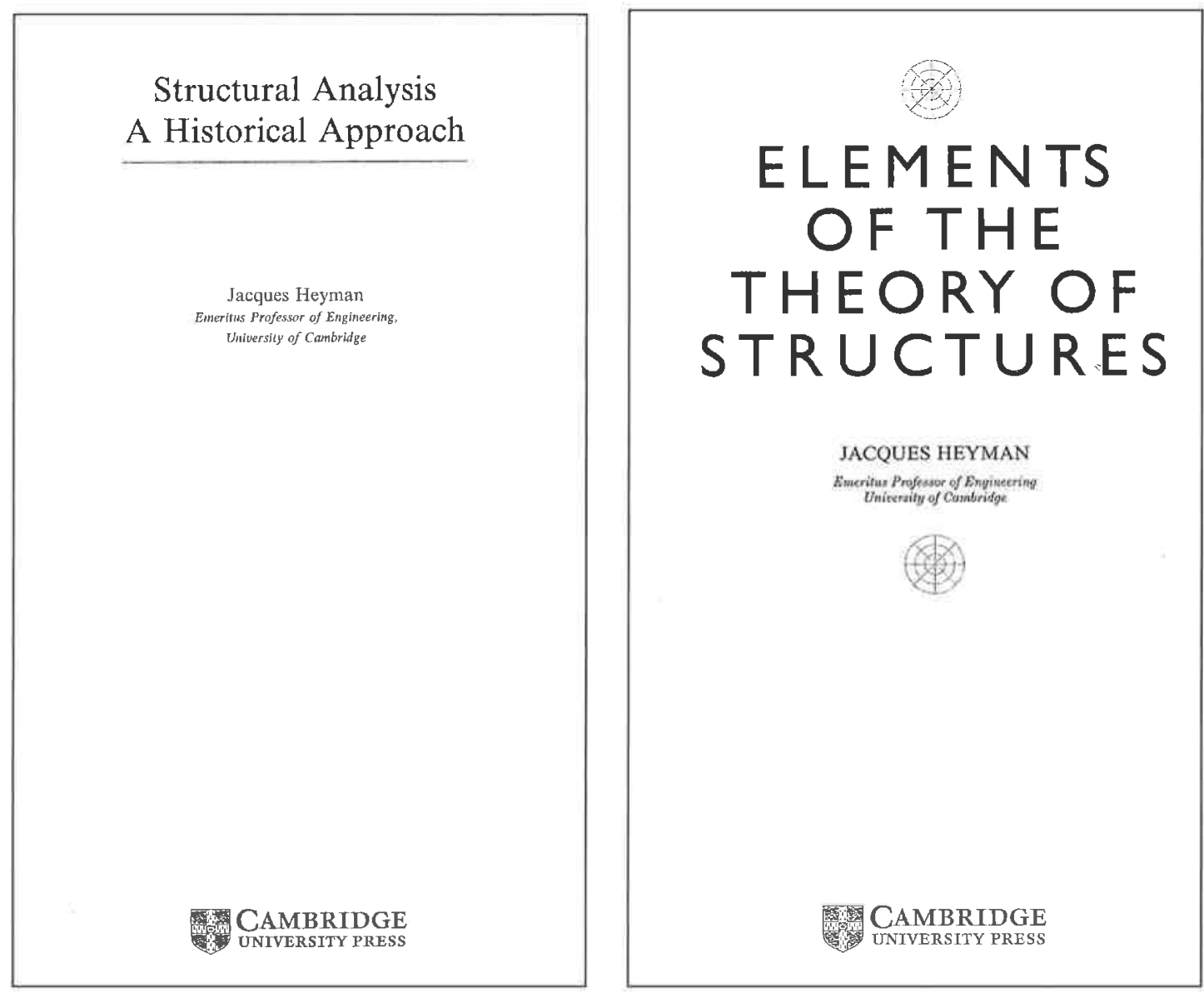

Bild 12. Titelseiten zweier Bücher Heymans

Fig. 12. Title pages of two books from Heyman 
Diskussionen über die Statik gotischer Gewölbe und Kathedralen, die unter anderem Viollet-le-Duc, Ungezwitter, Mohrmann und Abraham auf den Plan gerufen hatten, zu einem Abschluß gebracht werden. Viele Ingenieure haben die tiefgehende Bedeutung und die praktischen Konsequenzen von Jacques Heymans Einsichten jedoch noch nicht erkannt und wenden auch weiterhin komplizierte Computerprogramme zur Berechnung von Konstruktionen aus Mauerwerk an.

Abschließend gilt es noch darauf hinzuweisen, daß Jacques Heyman ein international anerkannter Gelehrter auf dem Gebiet der Geschichte der Bautechnik und der Baustatik ist. Zu seinen Veröffentlichungen gehören Biographien herausragender Persönlichkeiten des Bauingenieurwesens. Insbesondere sein 1972 veröffentlichtes Buch über Coulombs Denkschrift zur Baustatik wurde zu einem Modell für diese Art von Studie [8.58]. Erwähnenswert sind weiterhin seine Arbeiten über Robert Hooke, Pierre Couplet, Christopher Wren u. a. Auch der Entwicklung der Historiographie der Baustatik verlieh er entscheidende Impulse. So erörterte er in seinem Buch „Structural Analysis, a Historical Approach" (Bild 12) [8.73] erstmals die Geschichte der Konstruktionstheorie unter Berücksichtung der Beiträge aus dem Bereich der Plastizitätstheorie. Damit vervollständigte Heyman frühere Ansätze von Todhunter und Pearson, Timoshenko, Truesdell und Benvenuto u. a., die lediglich auf die Geschichte der Schnittkraftberechnung nach der Elastizitätstheorie und auf die Festigkeitslehre eingegangen waren. Ohne Berücksichtigung der Plastizitätstheorie ist der aktuelle Stand der Baustatik jedoch nicht nachzuvollziehen. Heymans Buch „Elements of the Theory of Structures" (Bild 12) [8.71] kann getrost als Prolegomenon einer Historischen Technikwissenschaft auf dem Gebiete der Baustatik gelesen werden; in souveräner Weise gelingt ihm dort eine Synthese zwischen Logischem und Historischem und weist nach, wie die geschichtliche Erkenntnis den technikwissenschaftlichen Erkenntnisprozeß zu befruchten vermag.

Jacques Heyman verkörpert die besten Qualitäten eines humanistischen denkenden und handelnden Bauingenieurs. Neben seiner Kompetenz und Originalität im Bereich hochkomplexer theoretischer Studien brachte er zeitlebens auch der Geschichte der Architektur und des Bauingenieurwesens großes Interesse entgegen. In einer Welt, in der Spezialisierung auf eng eingegrenzte Fachbereiche unumgänglich zu sein scheint, gelang es ihm, die Bedeutung allgemeiner Theorien für das Verständnis spezieller Phänomene und die Notwendigkeit historischer Studien für die Beurteilung der alktuellen Situation - nicht zuletzt auf dem Gebiet der Bautechnik - aufzuzeigen.

\section{Schlußbemerkungen}

William Addis hat den Einbruch des plastizitätstheoretisch in den elastizitätstheoretisch begründeten Tragwerksentwurf als Paradigmenwechsel im Sinne von Thomas S. Kuhns [42] beschrieben [43]. Während in Großbritannien dieser Paradigmenwechsel schon 1956 vollzogen war und alsbald andere Länder folgten, avancierte die Traglasttheorie im Stahlbau in der Bundesrepublik Deutschland erst nach Schaffung der neuen Stahlbau-Grundnormen DIN 18800 in der Zeit von 1981 bis 1990 - freilich mit dem konsequenten Über- gang vom Konzept der zulässigen Spannungen zum Konzept mit Teilsicherheitsbeiwerten - zum integralen Bestandteil der Bemessungspraxis. So schrieben die Autoren und Herausgeber der Erläuterungen zu DIN 18800 Teil 1 bis Teil $4 \mathrm{im}$ Vorwort: „Als Fortschritt ist die heute weitgehend problemlose Berechnung von Stabwerken nach Elastizitäts- und Plastizitätstheorie, also auch mit planmäßiger Nutzung plastischer Reserven des Stahls - dies auch nach Theorie II. Ordnung - zu nennen" [44, S. I]. Mit der Entzauberung der Schlauheit des Materials hörte auch die Rede des praktischen Statikers von der Schlauheit des Materials auf.

\section{Verzeichnis der wissenschaftlichen Veröffentlichungen Heymans auf dem Gebiet des Stahlbaus und der Baustatik}

1. Approximate methods of calculating collapse loads of stanchions bent in double curvature. Welding Research, Vol. 2, no. 4, p. 635, August 1948 (mit J. W. Roderick).

2. Tests on miniature portal frames. The Structural Engineer, Vol. 28, no. 6, p. 139, June 1950 (mit J. F. Baker).

3. Extensions of the simple plastic theory to take account of the strain-hardening range. Proc. I. Mech. E., Vol. 165, p. 189, 1951 (mit J. W. Roderick).

4. The limit design of space frames. Journ. Appl. Mech., Vol. 18, no. 2, p. 157, June 1951

5. The plastic design of beams and plane frames for minimum material consumption. Quart. Appl. Math., Vol. 8, no. 4, p. 373, January 1951.

6. Elasto-plastic stresses in transversely-loaded beams. Engineering, Vol. 173, pp. 359, 389. March 21 and 28, 1952.

7. The limit design of a transversely-loaded square grid. Journ. Appl. Mech., Vol. 19, no. 2, p. 153, June 1952.

8. Plastic analysis and design of steel-framed structures. Fourth Congress I.A.B.S.E., p. 95. Cambridge und London 1952.

9. Approximate methods in the limit design of structures. Proc. First U.S. Congress of Applied Mechanics, 1952.

10. Plastic design of plane frames for minimum weight. The Structural Engineer, Vol. 31, no. 5, p. 125, May 1953.

11. The plastic theory of structural design. The Engineering Journal (Engineering Institute of Canada), Vol.36, no. 12, p. 1603, December 1953 (mit D. T. Wright und V. L. Dutton).

12. The plastic design of grillages. Engineering. Vol. 176, p. 804, December 25, 1953.

13. Plastic design of plate girders with unstiffened webs. Welding and Metal Fabrication, Vol. 22, no. 7, p. 265, July 1954 (mit V. L. Dutton).

14. Full-scale loading tests on a welded plate-girder floor system. Conference on the Correlation between Calculated and $\mathrm{Ob}$ served Stresses and Displacements in Structures. Instn civ. Engrs, p. 156, September 1955 (mit B. E. S. Ranger).

15. Ossatures métalliques dans le stade plastique. Schweizer Archiv, Vol. 22, no. 7, p. 213, July 1956 (mit J. F. Baker).

16. Experimental verification of the strengths of plate girders designed in accordance with the revised British Standard 153: tests on full-size and on model plate girders. Proc. Instn civ. Engrs, Part III, Vol. 5, p. 462, August 1956 (mit E. Longbottom).

17. Plastic design of single-storey frames. British Welding Journal, Vol. 3, p. 332, August 1956.

18. Design problems. British Welding Journal, Vol. 3, p. 372, August 1956 (mit M. R. Horne).

19. The steel skeleton, Vol. 2: Plastic Behaviour and design. Cambridge: Cambridge University Press 1956 (mit J. F. Baker und $M$. R. Horne)

20. The study of plasticity in Russia. Nature, Vol. 179, p. 444, March 2, 1957. 
21. Plastic design of portal frames. Cambridge: Cambridge University Press 1957. Polnische Ausgabe: Projektowanie ram portalowych metoda nosnosci granicznej. Warschau: Arkady 1961.

22. Tests on I-section stanchions bent about the major axis. British Welding Journal, Vol. 4, p. 373, August 1957.

23. Plastic design of new laboratory block for B.W.R.A. British Welding Journal, Vol. 4, p. 488, October 1957.

24. Plastic design of pitched-roof portal frames. Proc. Instn civ. Engrs., Vol. 8, p. 119, 1957.

25. Rotating disks - Insensitivity of design. Proc. $3^{\text {rd }}$ U.S. Congress Appl. Mech., p. 551, 1958.

26. Plastic design of rotating discs. Proc. I. Mech. E., Vol. 172, p. 531, 1958 (mit dem James Clayton-Preis ausgezeichnet).

27. Minimum weight of frames under shakedown loading. Proc. A.S.C.E., Vol. 84, no. EM4, October 1958.

28. Automatic minimum weight design of steel frames. Journal of the Franklin Institute, Vol. 266, no. 5, p. 339, November 1958 (mit W. Prager).

29. Automatic design of steel buildings. Engineering, Vol. 186, p. 834, December 25, 1958.

30. Automatic analysis of steel-framed structures under fixed and varying loads. Proc. Instn civ. Engrs., Vol. 12, p. 39, 1959.

31. Limit design of framed structures. Engineering, Vol. 187, p. 275, February 27, 1959.

32. Limit design of framed structures made of imperfectly plastic materials. Bulletin de l'Académie Polonaise des Sciences, Vol. 7, no. $2-3$, p. 157 , 1959. Siehe auch: Non-homogenity in elasticity and plasticity, pp. 251-257. London: Pergamon Press 1959.

33. Inverse design of beams and grillages. Proc. Instn civ. Engrs, Vol. 13, p. 339, 1959.

34. On the absolute minimum weight design of framed structures. Quarterly J. Mech. Appl. Math., Vol. 12, part 3, p. 314, 1959.

35. Progress in plastic design, Plasticity. Proceedings of the second symposium on naval structural mechanics, pp. 511-535. London: Pergamon Press 1960.

36. On the miminum-weight design of a simple portal frame. Int. J. Mech. Sci., Vol. 1, p. 121, 1960.

37. An approach to the design of tall steel buildings. Proc. Instn civ. Engrs., Vol. 17, p. 431, 1960.

38. On the estimation of deflexions in elastic-plastic framed structures. Proc. Instn civ. Engrs., Vol. 19, p. 39, 1961.

39. The plastic design of latticed portal frames. The Structural Engineer, Vol. 39, p. 318, October 1961 (mit B. H. Fisher und L. G. Jaeger).

40. The calculation of steel frames. Progress in Applied Mechanics, p. 263. Macmillan 1963.

41. The plastic design of tall steel buildings. The design of high buildings, p. 192. Hong Kong University Press 1963.

42. Beams and framed structures. London: Pergamon Press 1964. Zweite Auflage 1974. Spanische Ausgabe: Vigas y porticos: Madrid: Juan de Herrera 2002.

43. A plastic composite design. Proc. Instn civ. Engrs., Vol. 32, p. 198, 1965 (mit R. P. Johnson und J. C. H. Finlinson).

44. Multi-storey welded steel frames. Building Materials, Vol. 26, no. 4 , p. $45,1966$.

45. Plastic theory of design for structures. Engineering, Vol. 201, p. 745, April 15, 1966.

46. Plastic theory and design, Chapter 23. In: The Steel Designers' Manual, 3rd Edition, London 1966 (mit J. F. Baker).

47. Plastic design in Britain, Publications. International Association for Bridge and Structural Engineering, Vol. 26, p. 31, 1966 (mit J. F. Baker).

48. The repeated loading of ductile steel structures. Proc. Instn civ. Engrs., Vol. 34, p. 477, 1966 (mit B. D. Threlfall).

49. A fully rigid multi-storey welded steel frame. The Structural Engineer, Vol. 44, p. 435, December 1966 (mit J. C. H. Finlinson und R. P. Johnson).

50. Bending moment distributions in collapsing frames. Engineering Plasticity. Cambridge, 1968.
51. The plastic design of braced multi-storey frames. 8th Congress I.A.B.S.E., New York, pp. 501-510, 1968.

52. Engineering plasticity (Papers for a conference held in Cambridge, March 1968). Cambridge: Cambridge University Press 1968 (Herausgeber zusammen mit F. A. Leckie).

53. Shakedown analysis: the design of a $275 \mathrm{kV}$ switchhouse. The Structural Engineer, Vol. 46, no. 4, p. 97, April 1968 (mit R. P. Johnson, P. P. Fowler und I. P. Gillson).

54. The simple plastic bending of beams. Proc. Instn civ. Engrs., Vol. 41, p. 751, 1968.

55. Plastic design of frames, Vol. 1. Cambridge: Cambridge University Press 1969 (mit Sir John Fleetwood Baker). 1980 als Paperback erschienen.

56. The full plastic moment of an I-beam in the presence of shear force. J. Mech. Phys. Solids, Vol. 18, pp. 359-365, 1970.

57. Plastic design of frames, Vol. 2. Cambridge: Cambridge University Press 1971.

58. Coulomb's Memoir on Statics: An essay in the history of civil engineering. Cambridge: Cambridge University Press, 1972. Nachgedruckt London: Imperial College Press 1997. Italienische Ausgabe: Il saggio di Coulomb sulla statica. Benevento (Hevelius Edizioni) 1999.

59. The significance of shakedown loading. 9th Congress I.A.B.S.E., Amsterdam, 1972.

60. Simple plastic theory applied to soil mechanics. Proc. Symp. Plasticity and Soil Mechanics, pp. 161-172, Cambridge, 1973.

61. Plastic design and limit state design. The Structural Engineer, Vol. 51, p. 127, 1973.

62. Overcomplete mechanisms of plastic collapse. Journal of Optimization Theory and Applications, Vol. 15, p. 27, 1975.

63. Les principes du calcul plastique, Les méthodes d'optimisation dans la construction. pp. 85-109. Paris: Editions Eyrolles 1975.

64. Equilibrium of shell structures. Oxford: Oxford University Press 1977.

65. Evolution des méthodes de calcul des structures. In: Evolution et théories modernes en élasticité et plasticité. p. 297. Paris 1979.

66. Elements of stress analysis. Cambridge: Cambridge University Press 1982

67. The masonry arch. Chichester; Ellis Horwood 1982.

68. The development of plastic theory 1936-48: some notes for a historical sketch. In: Instability and plastic collapse of steel structures (Hrsgn. V. L. J. Morris). London: Granada 1983.

69. The stone skeleton. Cambridge: Cambridge University Press 1995. Spanische Ausgabe: El esqueleto de piedra, Madrid: CEHOPU/ Juan de Herrera 1999.

70. Estructuras de fábrica. Madrid: Juan de Herrera 1995 (eine Sammlung von 28 Aufsätzen über das Tragverhalten von Mauerwerk-Konstruktionen ins Spanische uibersetzt).

71. Elements of the theory of structures. Cambridge: Cambridge University Press 1996

72. Arches, vaults and buttresses. Aldershot: Variorun 1996 (eine Sammlung von 26 Aufsätzen über das Tragverhalten von Mauerwerk-Konstruktionen).

73. Structural analysis: a historical approach. Cambridge: Cambridge University Press 1998. Spanische Ausgabe: Análisis de estructuras: Un estudio histórico. Madrid: Juan de Herrera 2004.

74. The science of structural engineering. London: Imperial College Press 1999. Spanische Ausgabe: La ciencia de las estructuras. Madrid: Juan de Herrera 2001.

\section{Literatur}

[1] Baker, J. F.: The steel skeleton, Vol. 1. Elastic behaviour and design. Cambridge: Cambridge University Press 1954.

[2] Baker; J. F., Horne, M. R., Heyman, J.: The steel skeleton, Vol. 2. Plastic behaviour and design. Cambridge: Cambridge University Press 1956. 
[3] Heyman, J.: Plastic design of portal frames. Cambridge: Cambridge University Press 1957.

[4] Huerta, $S$. (Ed.): Essays in the history and theory of structures. In honour of Jacques Heyman. Madrid: Instituto Juan de Herrera 2005.

[5] Kurrer; K.-E.: Geschichte der Baustatilk. Berlin: Ernst \& Sohn 2002

[6] Kazinczy, G. च.: Versuche mit eingespannten Trägern (in Ungarisch). Betonszemle 2 (1914), S. 68-71, S. 83-87 u. S. 101104.

[7] Kaliszky, S.: Gábor Kazinczy 1889-1964. Periodica Polytechnica (Civil Engineering) 28 (1984), S. 75-93.

[8] Gebbeken, N.: Eine Fließgelenktheorie höherer Ordnung für räumliche Stabtragwerke (zugleich ein Beitrag zur historischen Entwicklung). Mitteilungen des Institutes für Statik der Universität Hannover, hrsgn. v. H. Rothert. Hannover: Insti tut für Statik der Universität Hannover 1988.

[9] Kist, N. C.: Die Zähigkeit des Materials als Grundlage für die Berechnung von Brücken, Hochbauten und ähnlichen Konstruktionen aus Flußeisen. Der Eisenbau 11 (1920), H. 23, S. $425-428$.

[10] Grüning, M.: Die Tragfähigkeit statisch unbestimmter Tragwerke aus Stahl bei beliebig häufig wiederholter Belastung. Berlin: Springer 1926.

[11] Kurrer, K.-E.: Hermann Maier-Leibnitz (1885-1962): Wegbereiter des Industriebaus der klassischen Moderne. Stahlbau 74 (2005), H. 8, S. 623-634.

[12] Maier-Leibnitz, H.: Beitrag zur Frage der tatsächlichen Tragfähigkeit einfacher und durchlaufender Balkenträger aus Baustahl St 37 und Holz. Die Bautechnik 6 (1928), H. 1, S. 1114 u. H. 2, S. 27-31.

[13] Mohr; O.: Beitrag zur Theorie der Holz- und Eisen-Constructionen. Zeitschrift des Architekten- und Ingenieur-Vereins zu Hannover 6 (1860), H. 2/3, S. 323-346 u. H. 4, S. 407-442.

[14] Maier-Leibnitz, H.: Versuche mit eingespannten Balken von I-Form aus Baustahl St 37. Die Bautechnik 7 (1929), H. 20, S. $313-318$

[15] Fritsche, J.: Die Tragfähigkeit von Balken aus Stahl mit Berücksichtigung des plastischen Verformungsvermögens. Der Bauingenieur 11 (1930), H. 49, S. 851-855, H. 50, S. 873-874 u. H. 51, S. $888-893$

[16] Girkmann, K.: Bemessung von Rahmentragwerken unter Zugrundelegung eines ideal plastischen Stahles. In: Sitzungs berichte der Akademie der Wissenschaften in Wien, math.naturw. Klasse Abt. IIa, 140. Band, H. 9 u. 10. Wien 1931.

[17] Girkmann, K.: Über die Auswirkung der „Selbsthilfe" des Baustahls in rahmenartigen Stabwerken. Der Stahlbau 5 (1932), H. 16, S. 121-127.

[18] Rothert, H., Gebbeken, N.: Zur Entwicklung des Traglastverfahrens. In: Festschrift Professor Dr.-Ing. Heinz Duddeck zu seinem sechszigsten Geburtstag, hrsgn. v. J. Scheer, H. Ahrens u. H.-J. Bargstädt, S. 15-42. Berlin: Springer 1988.

[19] Kann, F.: Rechnerische Untersuchung über die Größe des Fließbereiches in stählernen Durchlaufbalken unter Berücksichtigung des Momentenausgleiches. Der Stahlbau 5 (1932), H. 14, S. 105-109.

[20] Kazinczy, G. v.: Statisch unbestimmte Tragwerke unter Berücksichtigung der Plastizität. Der Stahlbau 4 (1931), H. 5 , S. 58-59.

[21] Kazinczy, G. v.: Die Weiterentwicklung der Plastizitätslehre. Technika (1931), H. 5 bis 7

[22] Stüssi, F., Kollbrunner, C. F.: Beitrag zum Traglastverfahren. Die Bautechnik 13 (1935), H. 21, S. 264-267.

[23 Polónyi, S.: Sicherheit - sich absichern. Bautechnik 72 (1995), H. 3, S. 199-205.

[24] Maier-Leibnitz, H.: Versuche zur weiteren Klärung der Frage der tatsächlichen Tragfähigkeit durchlaufender Träger aus Baustahl St 37. Der Stahlbau 9 (1936), H. 20, S. 153-160.
[25] Fritsche, J.: Grundsätzliches zur Plastizitätstheorie. Der Stahlbau 9 (1936), H. 9, S. 65-68.

[26] Stüssi, F.: Gegen das Traglastverfahren. Schweizerische Bauzeitung 80 (1962), H. 4, S. 53-57.

[27] Symonds, P. S., Neal, B. G.: The Interpretation of Failure Loads in the Plastic Theory of Continuous Beams and Frames. Journal of the Aeronautical Sciences 1952, Vol. 19, Nr. 15, pp. 15-22.

[28] Reckling, K.-A.: Plastizitätstheorie und ihre Anwendung auf Festigkeitsprobleme. Berlin: Springer 1967.

[29] Baker, J. F.: A new method for the design of steel building frames. In: Abhandlungen der Internationalen Vereinigung für Brückenbau und Hochbau (IVBH). Vierter Band, hrsgn. v. L. Karner u. M. Ritter, S. 113-129. Zürich: Generalselkretariat der IVBH 1936.

[30] Baker, J. F.: The design of steel frames. The Structural Engineer, 1949, Vol. 27, pp. 397-431.

31] Neal, B. G., Symonds, P. S.: The calculation of collapse loads for framed structures. Journal of the Institution of Civil Engineers, 1950-51, Vol. 35, p. 21.

[32] Thürlimann, B.: Grundsätzliches zu den plastischen Berechnungsverfahren. Schweizerische Bauzeitung 79 (1961), H. 48, S. 863-869 u. H. 49, S. 877-881.

[33] Thürlimann, B.: Richtigstellungen zum Aufsatz "Gegen das Traglastverfahren“. Schweizerische Bauzeitung 80 (1962), S. $123-136$.

[34] Massonnet, C.: Kritische Betrachtungen zum Traglastverfahren. VDI-Z 105 (1963), Nr. 23, S. 1057-1063.

[35] Massonnet, C.: Die europäischen Empfehlungen (EKS) für die plastische Bemessung von Stahltragwerken. Acier-StahlSteel 32 (1976), S. 146-156.

[36] Vogel, U.: Die Traglastberechnung stählerner Rahmentragwerke nach der Plastizitätstheorie II. Ordnung. Forschungshefte aus dem Gebiete des Stahlbaus Heft 15. Berlin: Springer 1965

[37] Calladine, Ch. (Ed.): Editorial. Masonry construction Structural Mechanics and other aspects. Dordrecht: Kluwer 1992.

[38] Gvozdev, A. A.: The determination of the value of the collapse load for statically indeterminate systems undergoing plastic deformation (in Russ.). In: Proceedings of the Conference on Plastic Deformations, December 1936, p. 19. Moskau/Leningrad: Akademiia Nauk SSSR 1938. In Englisch: International Journal of Mechanical Sciences, 1960, Vol. 1, pp. 322335.

[39] Greenberg, H. J., Prager, W.: On Limit Design of Beams and Frames. Trans. ASCE 117 (1951), pp. 447-484. Diskussion: Proc. ASCE 78 (1952), pp. 459-484

[40] Kazinczy, G. v.: Die Plastizität des Eisenbetons. Beton und Eisen 32 (1933), H. 5, S. 74-80.

[41] Prager, W: Probleme der Plastizitätstheorie. Basel: Birkhäuser 1955 .

[42] Kuhn, T. S.: Die Strukturwissenschaftlicher Revolutionen. Frankfurt/M.: Suhrkamp 1979.

[43] Addis, W.: Structural Engineering. The Nature of Theory and Design. Chichester: Ellis Horwood 1990.

[44] Lindner, J., Scheer, J., Schmidt, H.: Stahlbauten. Erläuterungen zu DIN 18800 Teil 1 bis Teil 4. Berlin: Beuth und Ernst \& Sohn 1993

\section{Autoren dieses Beitrages:}

Dr.-Ing. Karl-Eugen Kurrer,

Verlag Ernst \& Sohn, Bühringstraße 10,

Prof. Dr. Santiago Huerta,

Departamento de Estructuras,

Escuela Técnica Superior de Arquitectura de Madrid,

Avda. Juan de Herrera 4, E-28040 Madrid 